\title{
Applications of Bayesian Networks and Petri Nets in Safety, Reliability, and Risk Assessments: A review
}

\begin{abstract}
System safety, reliability and risk analysis are important tasks that are performed throughout the system lifecycle to ensure the dependability of safety-critical systems. Probabilistic risk assessment (PRA) approaches are comprehensive, structured and logical methods widely used for this purpose. PRA approaches include, but not limited to, Fault Tree Analysis (FTA), Failure Mode and Effects Analysis (FMEA), and Event Tree Analysis (ETA). Growing complexity of modern systems and their capability of behaving dynamically make it challenging for classical PRA techniques to analyse such systems accurately. For a comprehensive and accurate analysis of complex systems, different characteristics such as functional dependencies among components, temporal behaviour of systems, multiple failure modes/states for components/systems, and uncertainty in system behaviour and failure data are needed to be considered. Unfortunately, classical approaches are not capable of accounting for these aspects. Bayesian networks (BNs) have gained popularity in risk assessment applications due to their flexible structure and capability of incorporating most of the above mentioned aspects during analysis. Furthermore, BNs have the ability to perform diagnostic analysis. Petri Nets are another formal graphical and mathematical tool capable of modelling and analysing dynamic behaviour of systems. They are also increasingly used for system safety, reliability and risk evaluation. This paper presents a review of the applications of Bayesian networks and Petri nets in system safety, reliability and risk assessments. The review highlights the potential usefulness of the BN and PN based approaches over other classical approaches, and relative strengths and weaknesses in different practical application scenarios.
\end{abstract}

Keywords: Bayesian networks, Petri nets, Safety analysis, Reliability analysis, Risk assessment

\section{Introduction}

Safety critical systems underpin many of the advances in modern society and have become an integral part of our life. Use of such systems can be noticed in a wide range of industries such as the automotive, aerospace, nuclear, energy, medical, and process industries. However, our high reliance upon them also means that the failure of such systems has the potential to cause great harm to people and the environment. For this reason, the development of such systems requires a rigorous assessment of system behaviour to ensure that they possess a high level of dependability: the capability of avoiding failures that are more frequent and more severe than it is acceptable.

One of the key goals in developing safety-critical systems is to identify potential risks posed by such systems and then minimising the likelihood of these risks. To increase the dependability of systems, analysts have to understand the behaviour of systems, i.e., how they work and how they may fail. The dependability analysis process allows system analysts to complete the above tasks. Based on the results of the analysis, decision makers can determine necessary preventive and/or maintenance actions to minimise the likelihood of system failure. As systems become more complex, dependability analysis plays a vital role in the successful design and development of the system; they also become increasingly difficult.

Over the years, several approaches have gained popularity including Fault Tree Analysis (FTA) (Vesely et al., 2002), Hazard and Operability Analysis (HAZOP) (Dunjó et al., 2010), Failure Modes Effect and Criticality Analysis (FMECA) (US Department of Defense, 1980), and Bow-tie diagrams (de Dianous and Fiévez, 2006) etc. These approaches often simplify the system safety and reliability analysis processes by making assumptions about system behaviour. Such assumptions include: focusing only on the technical part 
of the system, single mode of operation for system, statistical independence of system components, binary states (failed or non-failed) of components, non-repairability of components, and availability of failure data. However, in practice all these assumptions are not valid. Modern systems frequently operate in multiple modes or phases, making them dynamic systems. For example, an aircraft can operate in take-off, flight, and landing phases. Similarly the fuel system of an aircraft can work in refuelling and consumption modes. Under non-repairability assumption, it is considered that once a component fails it remains in the failed state forever and is not repaired. However, in many practical situations, it is possible to repair a failed component to restore its functionality. Moreover, it is also considered that systems and components can either be in working or in failed state, ignoring any intermediate state. However, in practice, systems and components can operate in other intermediate states with degraded functionality or performance levels.

Dynamic behaviour gives rise to a variety of dynamic failure characteristics such as functional dependencies between events and priorities of failure events, and the analysis is further complicated as organizational and human factors may contribute to system failure (Leveson et al., 2009). Overall, various challenges arise in the analysis from the following factors (Weber et al., 2012):

1. Scale and complexity (Seong, 2008; Zio, 2009)

2. Number and types of failure modes components can have (Filieri et al., 2010; Pham, 1999, 2003)

3. Dependencies among system components (Dugan et al., 1990; Xing et al., 2011)

4. Number of states the system can be in (Bouissou and Pourret, 2003; Li and Zuo, 2008; Lisnianski and Levitin, 2003; Yingkui and Jing, 2012)

5. Temporal behaviour of the systems (Berthomieu and Diaz, 1991; Labeau et al., 2000; Palshikar, 2002; Kabir et al., 2017)

6. Effect of organizational and human errors on system failure (Davoudian et al., 1994; Swain and Guttmann, 1983)

7. Uncertainties in system behaviour and failure data (Hu et al., 2015a; Kabir, 2016; Nannapaneni and Mahadevan, 2016)

To overcome the limitations of the classical approaches in addressing the above challenges, classical approaches have been extended, e.g., in the case of FTA, with dynamic fault trees (DFTs) (Dugan et al., 1992) or Temporal Fault Trees (TFTs) (Palshikar, 2002; Walker, 2009). Moreover, Markov chains, simulations (e.g. Monte Carlo), Petri nets, and Bayesian networks have been applied in the field to address the limitations of classical approaches. Markov chains are limited to exponential distributions. This requirement may be too tight for modelling complex systems as Markov Chains can only be used for systems with exponentially distributed lifetime. Additionally, Markov chain based approaches suffer from the state-space explosion. Simulation is usually used when the state-space analysis is infeasible. Simulation approaches can work with both exponential and non-exponential distributions. However, simulation requires more memory and can take much longer to run. It cannot be exhaustive and is typically limited to a subset of state trajectories. Bayesian networks can avoid the state space explosion problem and generally can work with different types of lifetime distributions. They can also deal with uncertainty and can be used for robust probabilistic reasoning in the conditions of uncertainty. The main advantage of BNs over other existing approaches is their polyvalency, i.e., they can offer different functionality such as predictive and diagnostic analysis, model updating, and optimization, etc. In the recent years, Bayesian networks have increased in popularity as shown in (Mahadevan et al., 2001; Boudali and Dugan, 2005a; Langseth and Portinale, 2007). Sigurdsson et al. (2001) and Weber et al. (2012) reviewed the applications of Bayesian networks for dependability, risk analysis and maintenance areas until 2000 and 2008, respectively. Some developments from 2009 were also included in (Weber et al., 2012). Petri nets have been applied in wide varieties of areas. A survey of the application of Petri nets for freight logistics and transportation systems modelling could be found in (Cavone et al., 2017). A review of different industrial applications of PNs is available in (Zhou and Zain, 2016). The application of Petri nets for system safety, reliability, and risk assessment can be traced back to the 1980s (Beyaert et al., 1981; Geist et al., 1983; Leveson and Stolzy, 1987) and their popularity for system analysis is increasing due to their strong mathematical formalism and graphical view. In addition to addressing the limitations of classical approaches for safety and reliability analysis, Petri net models provide a one-to-one 
interface for other purposes such as formal specification and verification, which cannot be handled with other formalisms.

This paper presents a comprehensive review of the use of BNs and PNs in system safety, reliability and risk assessments. The rest of this paper is organized as follows. Section 2 describes the fundamentals of Bayesian networks and Petri nets. The applications of BNs and PNs in system safety, reliability and risk assessment are reviewed in sections 3 and 4, respectively. Discussion and future research directions are presented in section 5. Finally, concluding remarks are presented in section 6 .

\section{Background}

\subsection{Bayesian Networks}

Bayesian Networks (BNs) are directed acyclic graphs that represent a set of variables and their conditional dependencies (Pearl, 1988). A BN model contains a qualitative and a quantitative part. The qualitative part contains a directed acyclic graph and the quantitative part contains prior and conditional probabilities of $\mathrm{BN}$ nodes. In the acyclic graph, the nodes represent the random variables and are graphically represented as circles. On the other hand, directed arcs represent dependencies or cause-effect relations among the nodes. In a $\mathrm{BN}$, a node $X$ is said to be the parent of another node $Y$ if there exists an arc from node $X$ to node $Y$. Parent nodes have a direct effect on its child nodes and each child node $X_{i}$ has a conditional probability distribution defined as $\operatorname{Pr}\left\{X_{i} \mid\right.$ Parents $\left.\left(X_{i}\right)\right\}$, which quantifies the effect of the parents on the child node. If a node has no parent, then it is known as a root node, and if a node has no children, then it is a leaf node.

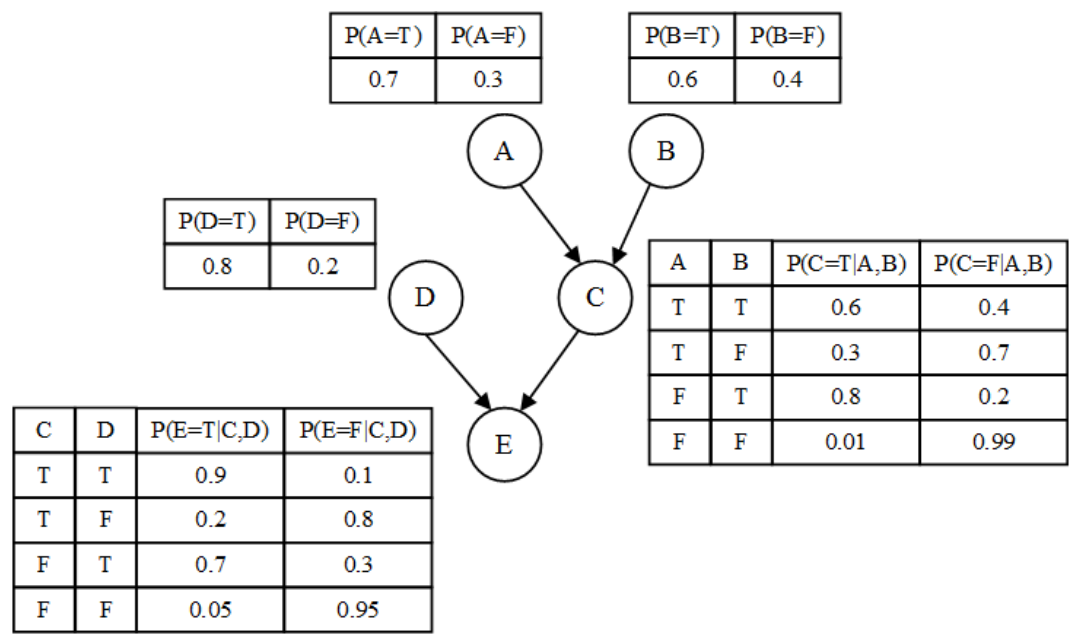

Figure 1: Example of a BN

A set of conditional independence statements are the main considerations when making BN models and conditional independence information can be obtained from a BN model by employing the rules of $d$-separation (Pearl, 1988). Two random variables $X$ and $Y$ are conditionally independent given a third random variable $Z$ if and only if they are independent in their conditional probability distribution given $Z$. Stated in a different way, $X$ and $Y$ are conditionally independent given $Z$ if and only if, given any value of $Z$, the probability distribution of $X$ is the same for all values of $Y$, and the probability distribution of $Y$ is the same for all values of $X$. Using the conditional independence assumptions of BNs, the joint probability distribution of a set of random variables $\left\{X_{1}, X_{2}, X_{3}, \cdots, X_{n-1}, X_{n}\right\}$ can be determined using a chain rule as explained in (Pearl, 1988):

$$
\operatorname{Pr}\left(X_{1}, X_{2}, X_{3}, \cdots, X_{n-1}, X_{n}\right)=\prod_{i=1}^{n} \operatorname{Pr}\left(X_{i} \mid \operatorname{Parent}\left(X_{i}\right)\right)
$$


BNs can provide a robust probabilistic method of reasoning under uncertainty and they are capable of combining different sources of information to provide a global assessment. In a BN model, both forward (predictive) and backward (diagnostic) analyses can be performed. The forward analysis of the model is done by following the directions of the network's arcs from the root nodes towards the leaf nodes. In this analysis, the information about the causes are used to obtain the new belief about the effects. On the other hand, the backward analysis of the BN model is performed by following the direction of the arcs in the opposite direction, i.e., reasoning from effects back to their causes. In this case, evidence is provided on the nodes representing unknown variables and based on this evidence new beliefs about the known variables are obtained and updated. The diagnostic analysis of BN model is performed using the Bayes' theorem. According to Bayes' theorem, if $A$ and $B$ are two random events and it is known that $B$ has occurred, then the posterior probability of the event $A$ occurring given that $B$ has occurred can be determined as:

$$
\operatorname{Pr}(A \mid B)=\frac{\operatorname{Pr}(B \mid A) \operatorname{Pr}(A)}{\operatorname{Pr}(B)}
$$

where $\operatorname{Pr}(A)$ and $\operatorname{Pr}(B)$ are the prior probability of event $A$ and $B$ respectively.

Classical BNs are particularly suitable for modelling systems in the static domain where the temporal behaviour of systems is not taken into account. However, classical BNs have been extended to model systems in dynamic domain as well. Application of $\mathrm{BNs}$ in dynamic domain has led to the formalisms like Dynamic Bayesian Networks (DBNs) (Nicholson, 1992; Murphy, 2002), Temporal Bayesian Networks $(\mathrm{TBN})($ Kanazawa, 1992), Dynamic Object Oriented Bayesian Networks (DOOBNs) (Weber and Jouffe, 2006), etc. Usually, a node in a BN represents either a discrete or a continuous variable. However, a hybrid $\mathrm{BN}$ (HBN) (Marquez et al., 2010) is a type of BN which can incorporate both discrete and continuous variables in a single $\mathrm{BN}$ model.

\subsection{Petri Nets}

Petri Nets (PNs) are a formal graphical and mathematical modelling tool which is appropriate for specifying and analysing the behaviour of complex, distributed and concurrent systems. A classical PN is a bipartite directed graph generally represented as six-tuple $N=\left(P, T, A, K, W, M_{0}\right)$, where:

- $P=\left\{p_{1}, p_{2}, p_{3}, \cdots, p_{n}\right\}$ is a finite set of places: a place is graphically represented by a circle.

- $T=\left\{t_{1}, t_{2}, t_{3}, \cdots, t_{m}\right\}$ is a finite set of transitions: a transition is represented by a rectangular bar.

- $A \subseteq(P \times T) \cup(T \times P)$ is a finite set of arcs from places to transitions or transitions to places.

- $K=\{1,2,3, \cdots\}$ is the capacity function of a place $p$, which defines number of resources a place can hold. Resources are represented as tokens (shown as dots).

- $W: A \rightarrow\{1,2,3, \cdots\}$ is the weight of an arc, which represents the number of tokens consumed from a place through an arc or number of tokens deposited to a place through an arc.

- $M_{0}: P \rightarrow\{1,2,3, \cdots\}$ is the initial marking of a PN model representing the number of tokens in each place of the model.

In a PN model, input places are known as the pre-conditions of a transition, which represent the conditions that need to be satisfied for the transition to be enabled and are connected to the transition through input arcs. Transition enabling conditions are defined as a pre-specified number of tokens in all the input places of the transition. On the other hand, output places are known as post-conditions of a transition, which represent the outcome of the transition and are connected to the transition through output arcs. On firing of a transition, a pre-specified number of tokens are consumed from the input places of that transition and a pre-specified number of tokens are deposited to the output places of the transition.

Although, classical PNs are easy to analyse, it requires many places and transitions to model the behaviour of moderately complex systems, which may give rise to a state explosion problem (Desrochers et al., 


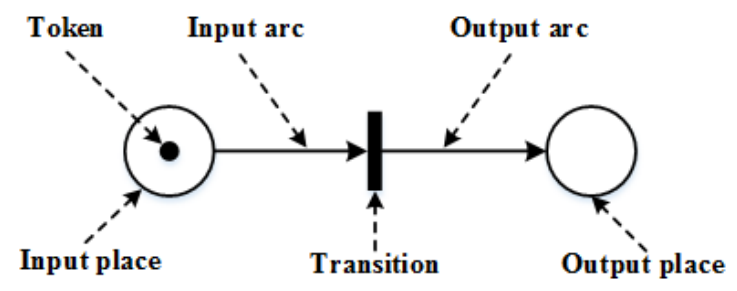

Figure 2: Example of a PN

2005). At the same time, it is problematic to model time-dependent behaviour using classical PNs. To overcome the above mentioned limitations, conventional PNs have gone through different modifications. Stochastic Petri Net (SPN) (Molloy, 1982) is one such extension of PNs that allows transition delays to be exponentially distributed. To allow both immediate and timed transitions in the same PN model, SPNs have been extended to Generalized Stochastic Petri Nets (GSPN) (Marsan and Chiola, 1987; Marsan et al., 1996). In GSPNs, timed transitions are usually represented graphically by white bars and fire after a random period of time. On the other hand, immediate transitions are graphically represented by black bars and they fire immediately after being enabled. Immediate transitions have priority over timed transitions, i.e., if an immediate and a timed transition are enabled at the same time, the immediate transition fires first.

Extended Stochastic Petri Nets (ESPNs) (Trivedi et al., 1984; Dugan et al., 1984) are another extension to SPNs, which allow transition delays to be defined as any arbitrary distribution. That means that nonexponentially distributed transitions are possible in ESPNs. On the other hand, Fluid Stochastic Petri Nets (FSPN) (Gribaudo et al., 2001a,b) allow modelling system behaviour with two classes of places: discrete places and fluid places. Discrete places may hold a discrete number of tokens and fluid places may hold a continuous quantity represented by a non-negative real number. The capability of PNs for system modelling has been enhanced in different other ways. One such enhancement is the inclusion of inhibitor arcs (Peterson, 1981) in the PN model. An inhibitor arc is represented as an arc that ends with a small circle instead of arrowhead. Inhibitor arcs can have a weight like normal arcs (Kleijn and Koutny, 2004) and they change the firing rule of transitions. If a place $p$ is connected to a transition $t$ through an inhibitor arc with weight $w$, then the transition $t$ will be enabled only if $p$ contains at most $w-1$ tokens. Moreover, when a transition fires, no token is consumed through an inhibitor arc, i.e., tokens in the inhibiting place remain zero. Using the property of the inhibitor arc, it is possible to verify non-occurrence of an event. There are different other variants of Petri nets available such as coloured Petri nets (CPNs) (Peterson, 1980; Jensen, 1981, 2013), Stochastic Well-formed nets (SWN) (Chiola et al., 1991, 1993), and predicate/transition nets (Genrich and Lautenbach, 1981). A detailed description of different types of PNs is out of scope of this paper. However, interested readers can find more information about Petri nets in (Reisig, 2012; Zurawski and Zhou, 1994; Peterson, 1977; Murata, 1989).

\section{Bayesian Networks in Safety, Reliability and Risk Assessments}

\subsection{Applications in Safety and Reliability Analysis}

Bayesian networks have been used in system safety and reliability analysis in two different ways. Firstly, they are used as standalone approaches. In such cases, system behaviour is directly modelled into a BN and all the relevant analyses are performed on the BN model. In the second scenario, BNs are used in association with other dependability analysis artefacts. In this case, generally dependability analysis artefacts such as fault trees are obtained first. After that, these artefacts are converted to BN models and all analyses are performed on these models.

\subsubsection{Applications as standalone approaches}

Early applications of BNs could be found in (Neil et al., 1996). A short review of applications of dynamic BNs for dynamic reliability assessment was presented in (Zahra et al., 2013). When BNs are used 
as standalone approaches, either predefined BNs are used for the analysis of a specific system or new BNs are created from scratch based on the knowledge about system under consideration. For example, in (Gran and Helminen, 2001), the authors used a BN model for reliability assessment of nuclear plants. BN models were used for the reliability analysis of power systems and military vehicles in (Yu et al., 1999; Daemi et al., 2012; Yongli et al., 2008; Hu et al., 2008) and (Neil et al., 2001), respectively. Network reliability has been studied in (Lynn et al., 1998) using a pre-defined BN.

When BNs are created from scratch, they can be created manually, (semi-)automatically from the existing data, or by combining manual and data-driven processes (Zhao et al., 2012). To create a BN for system reliability analysis, the information about the nodes, the association among the nodes and the strength of these associations must be known a priori. To help with the creation of a BN model, an expert with significant knowledge about the system is typically employed. Fig. 3 shows the steps required to create and utilise a BN model for safety and reliability analysis. Bouissou et al. (1999) proposed a method for hierarchical top-down construction of BNs combining evidence from different sources for reliability assessment of safety-critical systems.

To overcome the limitation of employing experts for creating BNs, and find associations between BN nodes, Doguc and Ramirez-Marquez (2009) proposed a method for automatic construction of a BN model of a system based on the historical data about the system without the intervention of experts. In this approach, associations between system components were identified using a data mining algorithm named K2. The same authors have performed similar research in (Doguc and Ramirez-Marquez, 2012) for reliability analysis of grid systems. Jiang et al. (2013) proposed a novel probabilistic model, called the hybrid relation model (HRM) for reliability evaluation of programmable logic controller (PLC) systems. HRM is a type of BN, which captures complex dependencies among PLC system components caused by the embedded software. Aslett et al. (2015) used the concept of survival signature (Coolen and Coolen-Maturi, 2013) for reliability evaluation of systems using BNs. A hybrid BN framework has been presented by Neil and Marquez (2012) to model the availability of renewable systems. Cai et al. (2012) have shown how a BN can be used for reliability evaluation of subsea blow-out preventer control system. Recently, Liu et al. (2015) proposed a method for developing a dynamic Bayesian network model for reliability analysis of subsea blowout preventer stack in the presence of common cause failures. In (Hänninen et al., 2014a; Eleye-Datubo et al., 2008) BNs have been used for safety and reliability analysis in the maritime and offshore industries. Hänninen (2014) reviewed the benefit and challenges of applications of Bayesian networks in maritime safety and accident analysis. Honari et al. (2009) showed how the Bayesian network can be used for the probabilistic reliability quantification of an $(r, s)$-out-of- $(m, n)$ : F system. In (Su et al., 2012), the application of DBN for reliability assessment of distribution system has been illustrated.

Although most applications target hardware reliability, methodologies have also been proposed for software systems. Fenton et al. (2008) reviewed the application of BNs for software defect prediction and reliability estimation. In (Bai, 2005), a new BN method called Markov Bayesian network has been proposed and applied for reliability assessment of software systems using data from their operational profile. In this approach, failure data of components were obtained from historical data and expert knowledge. At the same time, a dynamic Bayesian network based method has been proposed in (Roshandel et al., 2007) to assess the reliability of software systems at the architectural level by creating a stochastic reliability model of the system. This approach was developed for design time analysis of system where no information about the runtime behaviour of the system is available. The technique used a component-based strategy to evaluate the overall system reliability, i.e., the reliability of the system components is evaluated first and then the results are combined to estimate system-level reliability.

Reliability of systems with small probabilities are usually evaluated using Monte Carlo simulation. But Monte Carlo simulation based approaches are expensive in terms of execution time and memory requirement. To overcome these limitations, Cadini and Gioletta (2016) have proposed a method by exploiting Bayesian Monte Carlo (BMC) approach (Ghahramani and Rasmussen, 2003). Straub and Kiureghian (2010) proposed a computational framework called enhanced Bayesian network (eBN) by combining Bayesian networks and structural reliability methods (Ditlevsen and Madsen, 1996) for reliability estimation of engineering structures and infrastructures. Recently, Straub and Papaioannou (2014) proposed a method by combining structural reliability methods with rejection sampling strategy (Gilks and Wild, 1992) for calculating the 


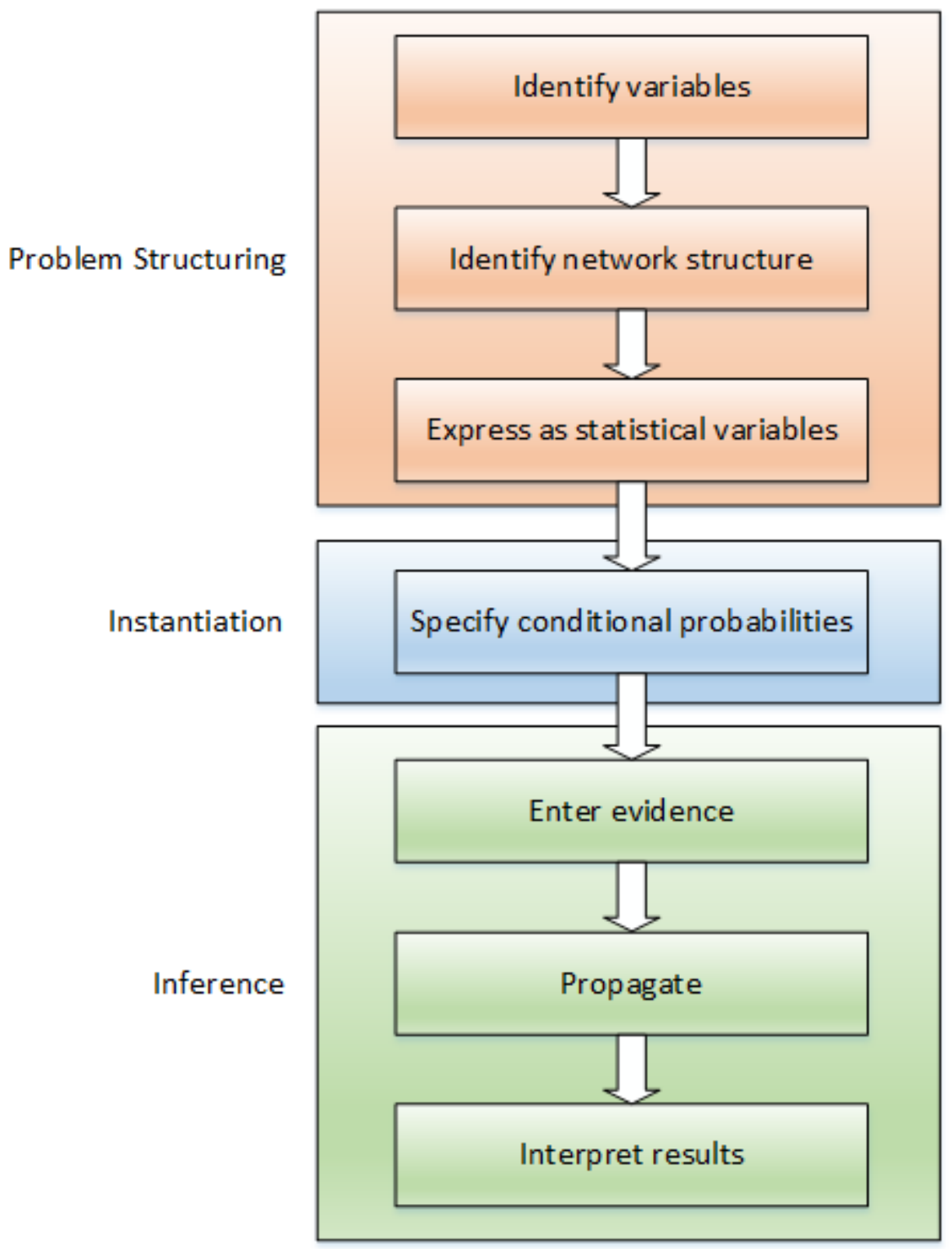

Figure 3: Steps necessary to build and use BNs for safety and reliability analysis (Sigurdsson et al., 2001)

posterior distribution of unknown parameters in a BN model.

Although most of the BN-based approaches rely on simulation to evaluate system reliability, Guan et al. (2012) proposed an analytical approach for reliability estimation using the Laplace method (Tierney and Kadane, 1986), Bayesian updating, and system response estimation given a reliability index. As this approach does not use simulations such as Monte Carlo or Markov chain Monte Carlo simulations, it is computationally more efficient, which makes this approach a superior option for real time application. Cai et al. (2015) also proposed a method combining classical and dynamic BNs for real time reliability evaluation. In this approach, classical $\mathrm{BN}$ is used for failure diagnosis, i.e., to identify the root causes of system failure and the DBN is used for the reliability analysis by performing predictive analysis on DBNs.

Most of the BN-based approaches estimate system reliability based on precise lifetime data of components. However, it is not always possible to obtain precise failure data of system components. BNs are inherently 
capable of handling random and non-random uncertainties, however, the issue of uncertainty has been explicitly addressed by different researchers. An overview of a few of such contributions is presented in Table 1.

Table 1: Overview of modelling aspects considered by BNs as part of standalone approaches

\begin{tabular}{|l|l|}
\hline Aspects considered & Contributions \\
\hline $\begin{array}{l}\text { Multi-state } \\
\text { systems }\end{array}$ & $\begin{array}{l}\text { Weber and Jouffe (2003, 2006), Bensi et al. (2013), Codetta-Raiteri et al. (2012), } \\
\text { Wilson and Huzurbazar (2007), Yontay and Pan (2016), Kang et al. (2015). }\end{array}$ \\
\hline $\begin{array}{l}\text { Dependency } \\
\text { among events }\end{array}$ & $\begin{array}{l}\text { Weber and Jouffe (2003, 2006), Musharraf et al. (2013),Salem et al. (2006), } \\
\text { Codetta-Raiteri et al. (2012), Su et al. (2014), Jiang et al. (2013), } \\
\text { Yontay and Pan (2016). }\end{array}$ \\
\hline \multirow{3}{*}{$\begin{array}{l}\text { Wncertainties } \\
\text { Human reliability }\end{array}$} & $\begin{array}{l}\text { Eleye-Datubo et al. (2008), Zhang et al. (2014), Görkemli and Ulusoy (2010), } \\
\text { Musharraf et al. (2013), Wu (2004, 2006), Fard et al. (2015), Ren et al. (2009). }\end{array}$ \\
\hline Eleye-Datubo et al. (2008), Mkrtchyan et al. (2016), Podofillini and Dang (2013), \\
Groth and Mosleh (2012), Baraldi et al. (2009), Musharraf et al. (2013, 2014), \\
Groth and Swiler (2013), Kim et al. (2006), Su et al. (2014), Li et al. (2012). \\
\hline
\end{tabular}

Wu $(2004,2006)$ addressed the issue of uncertain data in reliability analysis by combining fuzzy set theory with Bayesian networks. The method was used for the reliability analysis of production systems in (Görkemli and Ulusoy, 2010). Huang et al. (2006) used fuzzy set theory to address the issue of imprecise failure data and proposed an approach to determine multi-parameter lifetime distribution of components. In this approach, membership functions of the failure rate data were estimated using neural networks and genetic algorithm. Zhang et al. (2014) proposed a fuzzy set theory based BN approach for safety analysis in construction projects. Ibrahim and Beiu (2011) proposed a BN-based method for reliability evaluation of nano-scale CMOS circuits.

Human reliability analysis (HRA) is an important aspect that requires significant consideration while designing dependable systems. HRA is concerned with systematically identifying and analysing the causes and effects of human errors in system operation. Several approaches have been developed based on BNs for HRA and are listed in Table 1. A review of the applications and gaps in the use of BNs in HRA could be found in (Mkrtchyan et al., 2015). Recently, for HRA with limited judgement, the same authors have proposed methodologies in (Mkrtchyan et al., 2016) for building conditional probability tables of BNs. Groth and Swiler (2013) proposed a BN-based version of the widely used Standardized Plant Analysis Risk-Human Reliability Analysis (SPAR-H) (Gertman et al., 2005) method in the nuclear industry. This approach was aimed to close the gap between the HRA research and HRA practice in this industry. A BNbased method has been proposed by Podofillini and Dang (2013) to aggregate expert judgements on human error probabilities. This method can be used to determine the relationship between given performance conditions and the human error probability. As claimed by the authors, this approach can be applied to enhance the treatment of expert opinion in existing HRA methods such as SPAR-H, CESA (Reer et al., 2004; Reer and Dang, 2007), and HEART (Williams, 1986) etc. At the same time, Musharraf et al. (2013) have used BN to model the interdependency between human factors and associated actions during offshore emergency conditions. Multi-expert knowledge was used to address the issue related to the scarcity of data, and evidence theory was used to handle uncertainty and conflict associated with expert opinion.

\subsubsection{Applications as model-to-model transformation approaches}

The first application of BNs as part of model-to-model transformation approach could be found in (Torres-Toledano and Sucar, 1998). In this approach, Torres-Toledano and Sucar modelled the system reliability using a reliability block diagram (RBD). The RBD was then transformed into a BN model for probabilistic reliability evaluation. Further work on this approach is available in (Kim, 2011; Zhou et al., 
2006a; Mi et al., 2012). In (Kim, 2011), the author extended an RBD with general gates (RBDGG) using the concept of reliability graph with general gates (RGGG) (Kim and Seong, 2002). In order to quantify system reliability, RBDGG was transformed into BNs. While the previous approaches considered binary states of system components, the approach proposed in (Zhou et al., 2006a) considered multi-state systems. In addition to multi-state systems, common cause failure (CCF) is also considered in (Mi et al., 2012).

The most extensive application of BNs in system safety and reliability evaluation as model-to-model transformation approaches is by translating fault trees (FTs) into BNs. The pioneering work on system reliability analysis by translating FTs into BNs was performed by Bobbio et al. (2001). The process of mapping a FT into a BN is shown in Fig. 4. As seen in the figure, the mapping of a FT into a BN model is a one-to-one mapping process. The basic events of the FT are mapped to the root nodes of BN, the intermediate events (e.g., logic gates) are mapped into the internal nodes and the top event of the FT is mapped to a leaf node. The prior probabilities of the root nodes of the BN are calculated using the failure probabilities of the basic events they represent. On the other hand, the conditional probabilities of the internal nodes are populated based on the functional definitions of the logic gates they represent. As the outcome of the logic gates are deterministic, i.e. true or false, the entries in the conditional probability tables (CPTs) are either 0 or 1.

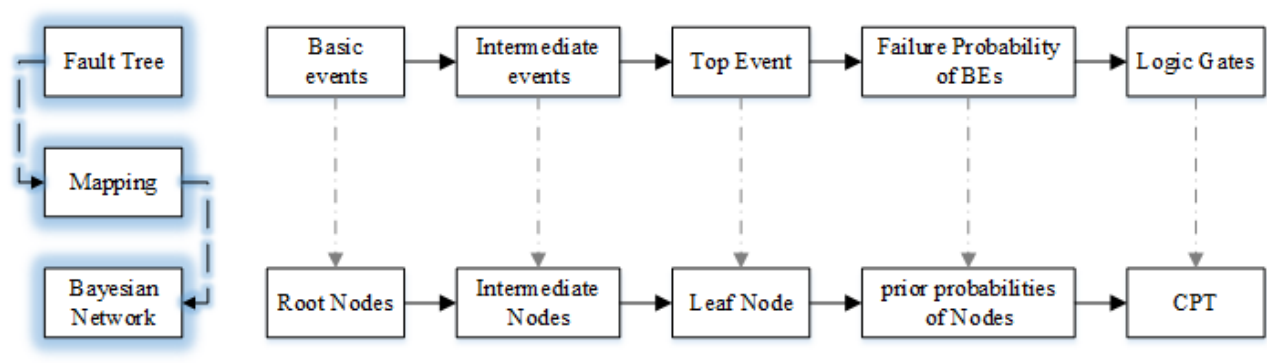

Figure 4: FT to BN conversion process

Once a $\mathrm{BN}$ is formed from of a FT model, predictive analysis is performed by following the arcs of the network. The result of predictive analysis usually consists of system reliability related information. On the other hand, diagnostic analysis can be performed by providing evidence about the system failure on the BN model. Based on this evidence the posterior probability of the root nodes of the BN can be calculated and updated. Evidence-based analysis is also used to determine the importance of the basic events in terms of their contributions to the occurrence of the system failure. In many areas, FTs have been translated to BNs for safety and reliability analysis of systems. For example, in (Martins and Maturana, 2013) FTA has been integrated with BNs for human reliability analysis in oil tanker operations. Some other applications include the reliability evaluation of steel construction projects (Leu and Chang, 2013), train control systems (Flammini et al., 2006; Su and Che, 2013), and power systems (Limin et al., 2002).

In most of the approaches mentioned earlier in this section, the prior probabilities of the component failures are considered as precise values. However, for large and complex systems, in many cases it is difficult to obtain a precise failure probability. The issue of epistemic uncertainty in failure data has been explicitly addressed using BNs. For instance, in (Simon et al., 2007, 2008), the authors combined evidence theory with BNs to address the issues of both random and epistemic uncertainties. The authors showed how the basic concepts of the Dempster Shafer theory (Shafer, 1976) can be implemented in BN tools for the treatment of epistemic uncertainty. Moreover, they also showed how diagnostic analysis can be performed to identify components that contribute more to the uncertainty.

In addition to classical fault trees, dynamic fault trees (DFTs) have also been probabilistically evaluated by translating them into Bayesian networks. Boudali and Dugan (2005a) proposed a method for reliability evaluation of dynamic systems by translating DFTs into discrete-time Bayesian networks (DTBNs). This approach can model complex behaviours of components and their interactions. It also alleviates the state space explosion problem met in the evaluation of DFTs using Markov chains. The qualitative translation 
process of DFTs to DTBNs is the same as the translation process of FTs to BNs, as seen in Fig. 4. The only difference is that, in addition to Boolean gates, dynamic gates are also translated into BNs. However, the quantitative part of the translation process, i.e., the generation of prior probabilities and conditional probabilities, are different from the static approach. Now, these probability values are generated by taking into account that a component can fail in different points in time and also by considering the dynamic behaviour of the logic gates. It is important to note that as this approach considers the discrete model of time, the granularity of temporal discretisation has to be decided prior to modelling. The accuracy and computational performance of this approach is dependant on the number of time intervals used for time discretisation. To overcome the issues with time discretisation, Boudali and Dugan (2006) proposed a method based on continuous time BNs. This approach is different from the DTBN-based approach in the sense that the variables in the BN are now continuous. More importantly, the probabilities (both prior and conditional) are expressed as probability density functions and the joint probability distribution is expressed as a joint probability density function instead of multi-dimensional tabular form. For this reason, this approach saves on memory, by not storing any probability tables, and can provide an exact closed form solution to the system reliability evaluation. Marquez et al. $(2008,2010)$ developed a BN-based approach where both discrete and continuous nodes are used in the same model and which allows the use of any parametric and empirical distributions for the time-to-failure of system components.

At the same time, Montani et al. (2005) proposed a concept of translating DFTs to dynamic Bayesian networks (DBNs) for dependability analysis of systems. They performed further research to propose the concept of a tool for automatic translation of DFTs to DBNs in (Montani et al., 2006b,a). Finally, they presented a complete tool named RADYBAN in (Montani et al., 2008) for automatic conversion of DFTs to a 2-time-slice BNs (2TBNs)(Weber and Jouffe, 2003). 2TBN is an instant-based approach, whereas the methodology proposed by Boudali and Dugan (2005a) was interval-based. The instant-based approach has been criticised for being too general or creating unnecessarily complex networks by repeating the same structure for each time instance. However, the 2TBN approach seems to overcome this limitation by modelling any time horizon using only two time slices. Portinale et al. (2010) further developed this work by introducing a repair box gate to enable the modelling of repairable systems. In (Codetta-Raiteri and Portinale, 2015), dynamic Bayesian networks were used for fault detection, identification, and recovery in autonomous spacecraft. Most recent contributions of BNs applications in safety and reliability by converting DFTs into BNs can be found in (Codetta-Raiteri, 2015; Codetta-Raiteri and Portinale, 2017). In (Codetta-Raiteri and Portinale, 2017), DFTs were evaluated by translating them into a Generalised Continuous Time Bayesian Network (GCTBN) (Codetta-Raiteri and Portinale, 2010), an extension of the CTBN defined by Nodelman et al. (2002). Unlike other variants of BNs, GCTBN can consider repairability of components during analysis. The GCTBNs were solved by converting them to GSPN models. For this reason, if the GCTBN contains many multi-state nodes, the GSPN-based analysis will suffer from state space explosion due to the consideration of large number of states.

RBDs and FTs are not the only artefacts that are transformed into BNs for system safety and reliability analysis. Different other artefacts such as bow-tie diagrams were translated to BNs in (Khakzad et al., 2013a; Abimbola et al., 2015) and FMEA was translated to BNs in (Chengshan and Yinghua, 2004). In addition to model-to-model transformation approaches, BNs have also been used in association with other dependability artefacts, where other models are not directly transformed into BNs. Instead, BN models are used in parallel with other models to improve the results. For instance, in Yang et al. (2008) have used BNs with FMEA and fuzzy set theory for risk prioritisation in FMEA. Weber et al. (2004) used a dynamic $\mathrm{BN}$ as an equivalent model to the Markov chain for reliability assessment of complex systems. On the other hand, Foulliaron et al. (2015) modelled semi-Markovian processes using a special type of DBN called Graphical duration model (GDM) (Donat et al., 2010) for reliability analysis of dynamic systems. In (Singh et al., 2001), BN has been used with UML diagrams for reliability evaluation of component based software systems. In this approach, system reliability is estimated using three models as: state-based models, pathbased models and additive models. In state-based models, predefined system architectures or architectures generated at run time are represented using control graphs (Gokhale et al., 1998). All possible execution paths that the system can follow during runtime are considered in the path-based models. Component failure data are used in the additive models (Xie and Wohlin, 1995) by considering the growth model. 
Table 2: BNs applications as model-to-model transformation approaches

\begin{tabular}{|c|c|c|}
\hline $\begin{array}{l}\text { Dependability } \\
\text { artefacts }\end{array}$ & $\begin{array}{l}\text { Methodological } \\
\text { contributions }\end{array}$ & $\begin{array}{l}\text { Applicative } \\
\text { contributions }\end{array}$ \\
\hline $\begin{array}{l}\text { Classical } \\
\text { fault trees }\end{array}$ & $\begin{array}{l}\text { Portinale and Bobbio (1999),Bobbio et al. (2001, 2003a), } \\
\text { Martins and Maturana (2013), Simon et al. (2007, 2008), } \\
\text { Franke et al. (2009), Wang et al. (2004, 2011a), } \\
\text { Xiaowei (2010),Xiaowei et al. (2008), Gu and Yang (2013), } \\
\text { Yazdi and Kabir (2017) }\end{array}$ & $\begin{array}{l}\text { Leu and Chang (2013), } \\
\text { Limin et al. (2002), } \\
\text { Lu et al. (2004), } \\
\text { Su and Che (2013) } \\
\text { Flammini et al. (2006) } \\
\text { Yin et al. (2008) } \\
\text { Zhou et al. (2006b) }\end{array}$ \\
\hline $\begin{array}{l}\text { Dynamic } \\
\text { fault trees }\end{array}$ & $\begin{array}{l}\text { Boudali and Dugan (2005a,b, 2006), Portinale et al. (2010) } \\
\text { Montani et al. (2005, 2006b,a, 2008), Mi et al. (2016), } \\
\text { Marquez et al. (2008, 2010), Codetta-Raiteri (2015), } \\
\text { Codetta-Raiteri and Portinale (2014, 2017), Li et al. (2015) } \\
\text { Khakzad et al. (2013c), Kabir et al. (2018a, 2014) }\end{array}$ & $\begin{array}{l}\text { Mi et al. (2016), } \\
\text { Codetta-Raiteri (2015) }\end{array}$ \\
\hline Bow-tie diagram & Khakzad et al. (2013a) & Abimbola et al. (2015) \\
\hline $\begin{array}{l}\text { Reliability } \\
\text { Block Diagram }\end{array}$ & $\begin{array}{l}\text { Torres-Toledano and Sucar (1998), Zhou et al. (2006a), } \\
\text { Kim (2011), Mi et al. (2012), Li et al. (2016a) }\end{array}$ & Ur et al. (2014) \\
\hline FMEA & Chengshan and Yinghua (2004) & Wang and Xie (2005) \\
\hline
\end{tabular}

\subsection{Applications in Risk Assessments}

A review of the applications of Bayesian inference for probabilistic risk assessment till 2007 was presented by Kelly and Smith (2009). Different aspects of Bayesian network-based risk assessment and decision analysis are described by Fenton and Neil $(2012,2014)$. The inference process of BN was integrated with traditional probabilistic risk assessment by Lee and Lee (2006) to consider the effects of evolutionary environmental conditions on nuclear waste disposal. Maglogiannis et al. (2006) introduced a BN-based method for risk analysis in patient health monitoring system. In this approach, they combined basic attributes of the CCTA Risk Analysis and Management Methodology (CRAMM) (Yazar, 2002) with the BN network to identify assets, potential threats and vulnerabilities of patient telemonitoring systems, and model dependencies between these events.

Like system safety and reliability analysis applications, different dependability artefacts such as event trees, fault trees, dynamic fault trees and bow-tie diagrams are translated into BNs for risk assessment. For example, Kalantarnia et al. (2009, 2010) and Rathnayaka et al. (2011) represented potential accident scenarios using event trees and utilised Bayes' theory as a means for dynamic risk assessment by helping to update the failure probabilities of the events in the event trees. Khakzad et al. (2012) used Bayes' theory with bow-tie (BT) model for dynamic risk assessment. The BT model combines fault tree and event tree to represent accident scenario including causes and consequences. This approach is suitable for real-time risk assessment. In the approach, the basic events failure probabilities are updated in real-time based on the observed parameters of the physical system components. On the other hand, Bayes' theorem is used to update the failure probability of safety barriers of the BT. Methodologies have been proposed for mapping bow-tie diagrams into BNs for dynamic risk assessment in (Ale et al., 2006, 2009; Khakzad et al., 2013a; Yuan et al., 2015). The approach proposed in (Khakzad et al., 2013a) was applied for the quantification of risk associated with offshore drilling operations in (Khakzad et al., 2013b; Abimbola et al., 2015). Bayesian network based risk assessment approaches have been widely used in process industries. Khakzad et al. (2011) compare the applications of fault trees and Bayesian networks in the safety risk analysis in process industries. Khakzad et al. (2013c) have proposed a method for converting DFTs into discrete-time BN for risk analysis in process industries. The authors proposed an algorithm called neutral dependency to avoid large, multi-dimensional conditional probability tables (CPTs). Using this algorithm, CPTs of PAND, AND, and OR gates are decomposed into two tables, each of which is smaller than the original CPTs. 
However, the inclusion of neutral dependency algorithm for complexity minimisation of CPTs makes this approach unsuitable for posterior/diagnostic analysis. Similar contribution has also been made by Barua et al. (2016). They proposed to use DFTs to model dynamic failure behaviour of chemical process plants and then convert the DFTs into DBNs for dynamic risk assessment. Khakzad et al. (2013d) proposed a new BN-based approach for probability estimation and failure propagation path determination of domino effects. Recently, Khakzad (2015) developed another Bayesian network methodology to model both the spatial and temporal evolutions of the cascading effects of a chain of accidents and to determine the most probable sequence of accidents in a process plant. (Hu et al., 2015b) proposed a dynamic Bayesian network based method for fault propagation studies in petrochemical process facilities. In this approach, firstly, a HAZOP study is performed to identify the faults, their causes and potential consequences. The causal relationships among different events are then modelled using DBN for risk assessment.

The modelling power of BNs has been utilised to model various aspects of maritime risk assessment such as the ship-ship collision or grounding occurrence (Akhtar and Utne, 2014; Hänninen et al., 2014b), accidents and their consequences (Antão et al., 2009; Kelangath et al., 2012; Zhang et al., 2013; Goerlandt and Montewka, 2014; Montewka et al., 2014). Hänninen and Kujala (2012) used Bayesian networks to identify the risk-influencing variables which significantly affect the occurrence of ship collision in the Gulf of Finland. Wu et al. (2015) proposed to integrate the interpretive structural modelling (ISM) method (Warfield, 1973) with BNs for risk assessment for operations in marine environments. To form the structure of the BN model and find the relationships among the BN nodes, this approach used the ISM method to systematically acquire information from experts. The prior probabilities of the BN nodes were determined based on expert opinions. The approach was applied to two offshore pipeline case studies to facilitate better risk assessment in associated maritime projects. When precise failure data are scarce, expert judgements are commonly used to obtain the prior probability of the BN nodes. However, there exists criticism about the subjectivity of the expert judgement. To address this issue, Li et al. (2014) developed a binary logistic regression method to obtain prior probabilities of the BN nodes by utilising the existing maritime accident data resources. Other recent contributions related to the risk assessment of oil and gas pipelines using Bayesian networks are available in (Aljaroudi et al., 2015; Li et al., 2016b; Wu et al., 2017; Li et al., 2017). Bayesian networks have been applied for conducting dynamic risk assessment of deepwater managed pressure drilling and under-balanced drilling operations by Bhandari et al. (2015).

Cai et al. (2013) proposed an algorithm to convert fault trees into a dynamic Bayesian network for quantitative risk assessment of human errors on offshore blowouts considering the repairability of events. In order to address the issue of scarce statistical failure data, Wang and Mosleh (2010) proposed a method based on BNs called QQBBN to incorporate both qualitative and quantitative information in the BN models. This approach is particularly useful when the risk assessment requires to take into account the influence of human and organizational factors. At the same time, Wang et al. (2011b) integrated fuzzy fault trees with BN for probabilistic risk assessment under conditions of uncertainty. Ren et al. (2009) combined fuzzy logic with BN for offshore risk analysis. In order to incorporate both qualitative and quantitative data in the risk assessment process under uncertainty, recently, John et al. (2016) developed a modelling approach combining BNs with fuzzy set theory. They applied this approach for resilience improvement of the seaport system by performing risk assessments.

Chin et al. (2009) proposed a BN-based method for risk assessment in new product development. In this approach, the risk factors in new product development project such as research and development risk, supply risk, production risk and product reliability were identified and their interrelationships were investigated first. After that, $\mathrm{BN}$ is used to model the relationships among those factors. A systematic probability generation algorithm was developed for prior and conditional probability generation of the nodes of the BN. However, for complex Bayesian network structures where a node has many parent nodes, and a large number of states is considered for a node, this approach creates difficulties in completing the large conditional probability tables. Duval et al. (2012) proposed a BN-based integrated risk assessment method for industrial risk assessment where all the potential risks such as risks associated with system models (e.g., functional and organizational), environmental conditions, human actions and maintenance strategy are globally covered in a single view. Li et al. (2010) proposed to construct BN models for flood risk assessment using domain knowledge and spatial data. Liu et al. (2012) used Bayesian network for health risk assessment using the 
example of road constructions. Recently, Brito and Griffiths (2016) proposed a Bayesian approach to predict the risk of losing Autonomous Underwater Vehicles (AUVs) during a mission in hazardous environments due to the deviations of operating conditions from its agreed nominal conditions. BNs have been used by Kabir Kabir et al. (2015a) to develop a model for evaluating risk of water main failure by considering different contributing factors such as hydraulic capacity, structural integrity, and water quality etc.

Similar to addressing human factors in system safety and reliability analysis applications, BNs have been used for human factor analysis in risk assessment. Trucco et al. (2008) proposed a method for integrating Human and Organisational Factors (HOF) into a risk analysis by combining fault tree analysis and Bayesian networks. In this approach, firstly, fault trees are used to represent the hazardous situations that may occur due to the failure of the systems. Afterwards, BN is used to modify the probability of the basic events of the fault tree by taking into account the effect of the human and organisational factors.

\section{Petri Nets in Safety, Reliability and Risk Assessments}

\subsection{Applications in Safety and Reliability Analysis}

Similar to BNs, PNs have also been used both as standalone approaches and as a part of model-to-model transformation approaches for system safety and reliability analysis. When used as standalone approaches, system behaviour is directly modelled using Petri nets and the model is then analysed to evaluate system safety and reliability. On the other hand, when used as part of model-to-model transformation approaches, system failure behaviour is captured first using a dependability analysis model as a fault tree. This model is then transformed into a PN model and the model is analysed to determine system safety and reliability.

\subsubsection{Applications as standalone approaches}

Petri nets have been used for the safety analysis of real-time safety critical software systems by Leveson and Stolzy (1987). In their approach, the nominal behaviour of systems is modelled using Timed Petri nets and the model is then extended by introducing failures and faults. This extended model is analysed for safety and criticality analysis of systems. It is important to note that only the severity of the hazards is used for safety analysis so the analysis remains qualitative. Dutuit et al. (1997) presented a PN-based approach which included qualitative analysis, i.e. determination of potential sequences of events that lead to system failure as well as reliability analysis with application to distributed processing systems and communication networks in (Kumar and Aggarwal, 1993; Muppala and Lin, 1996) and (Balakrishnan and Trivedi, 1996), respectively.

A tutorial has been provided in (Schneeweiss, 2001) showing how PNs can be used to model different reliability scenarios. Fig. 5 shows the typical steps that are required to create and analyse a PN model for safety and reliability analysis. Volovoi (2004) proposed a flexible and graphical modelling framework for reliability analysis of non-exponentially distributed data. The approach was demonstrated with several examples, including load sharing, shared pool of identical imperfectly repaired components, phased mission systems, and damage tolerant components.

Mura and Bondavalli (2001) used Markov Regenerative Stochastic Petri Nets to propose an analytical technique with low computational complexity for dynamic behaviour modelling and analysis of phased mission systems. Recently, Wu and Wu (2015) proposed an extended object-oriented Petri net (EOOPN) model for mission reliability analysis of the repairable phased mission system with common cause failures. Adamyan and He (2002a) used PNs to model sequence-dependant failure behaviour of manufacturing systems. Using the PN model, the potential sequences of events that can cause the system failure were identified and based on these information, safety and reliability are evaluated. Failure rates of system components are assumed to be exponentially distributed. To overcome this limitation the same authors proposed a method in (Adamyan and He, 2003) and (Adamyan and He, 2004), which allows the use of inhibitor arcs and loops in PN model. They also use fewer variables than in earlier work to reduce computational complexity. Sadou and Demmou (2009) proposed a PN based approach for temporal behaviour analysis of dynamic systems. Using PN, they derive the causes of reaching a feared (unexpected) scenario. Reliability is evaluated by identifying a set of minimal sequence of scenarios that can cause the system to go to the feared state. A 


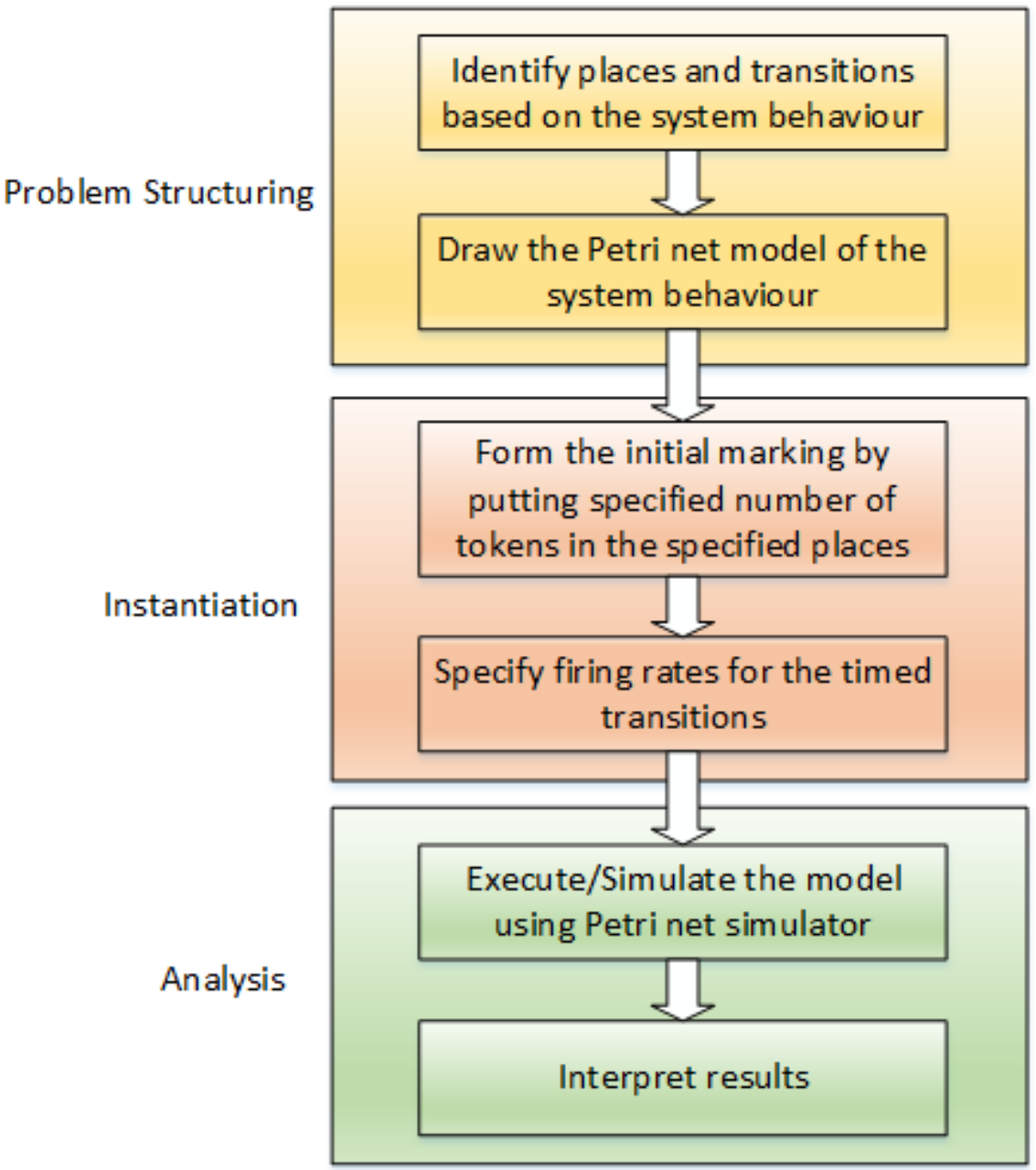

Figure 5: Typical steps for building and analysing PN models for safety and reliability analysis

reachability graph that is generated from PN to determine failure scenarios can easily lead to state space explosion. To avoid this problem the authors in (Sadou and Demmou, 2009) translate the reachability graph of a PN to equivalent linear logic sequents.

A model-based approach was proposed by Portugal et al. (2005) using Stochastic Petri nets (SPNs) for dependability analysis of controller area network in the presence of transient faults occurred during communication. Sachdeva et al. (2008) proposed a method based on PNs for reliability evaluation of the pulping system in the paper industry. Interactions between different active and standby units of the system were modelled using Stochastic Reward Nets. Monte Carlo simulation was then used to evaluate the reliability of the system. Signoret (2009) showed how SPNs can be used for dependability analysis and safety integrity level allocation problems. Application of SPNs for availability analysis of safety critical on-demand systems was presented in (Kleyner and Volovoi, 2010). The method proposed in this application takes into account different practical factors such as detectability of faults, duration of repairs, estimated down time, system age, etc. Zeng et al. (2012) used SPNs to model the behaviour of control networks in smart grid by considering cold and hot spare strategies. Transient and steady-state analysis were performed on the SPN models to evaluate reliability and availability of the smart grid system. A method was proposed to avoid the state-space explosion problem. Colored Petri net (CPN) has been used by Pinna et al. (2013) for safety and reliability analysis. A guideline was provided for hierarchical modelling of both deterministic 
and probabilistic features of system behaviour using CPN. Monte Carlo simulation was used for reliability evaluation.

Ionescu et al. (2006) have used GSPN for the reliability modelling of medium voltage distribution systems of nuclear power plants. GSPNs have also been used by Ghosh et al. (2017) for reliability modelling and analysis of phasor measurement units (PMUs) in power systems. Codetta-Raiteri and Bobbio (2005) used GSPN and FSPN for solving dynamic reliability problem. On the other hand, colored PN has been used by Németh et al. (2009) for primary-to-secondary leaking safety procedure verification of nuclear power plants. In order to deal with imprecise, uncertain information in the reliability analysis of repairable systems, Garg (2013) proposed a method combining PNs with intuitionistic fuzzy sets. PN is used for qualitative modelling of behaviour of systems. For quantitative analysis, failure rates and repair times of system components are represented by intuitionistic fuzzy numbers of triangular membership functions instead of crisp or classical fuzzy numbers.

PN has been used by Singh and Rajput (2016) for modelling and safety analysis of computer based safety critical systems. The same authors proposed another PN-based method in (Singh and Rajput, 2017) for dependability analysis of real-time systems. In this approach, they considered different properties such as non-liveness, deadlock, stability, etc., during dependability evaluation. A hierarchical approach was proposed by Chung and Chang (2011) to create a PN model for the purification operation in the Metal Organic Chemical Vapor Deposition (MOCVD) process. The model was then simulated to identify the effects of different component failure and external interferences. Hybrid Petri nets were used by Ghasemieh et al. (2016) to model the behaviour of waste water treatment facility. The survivability of the system was evaluated by using model checking on the PN model. Vasilyev et al. (2017) used hybrid Petri nets for reliability modelling of polymer electrolyte membrane (PEM) fuel cell systems. Li et al. (2016c) proposed a PN-based reliability modelling method when system reliability was evaluated considering the dependence of failure mechanism. Wieland et al. (2009) proposed a PN based model to calculate reliability data of polymer-electrolyte-membrane fuel cell stacks. The reliability data include the average lifetime of a single stack or the reliability of stacks of a whole fuel cell vehicle fleet within a given time. Zareiee et al. (2014) developed PN-based controller for safety analysis of discrete event systems. This controller prevents the system from entering any forbidden state by solving Integer Linear Programming problems. Singh et al. (2017) used PNs for safety modelling of gantry crane operations. Gonçalves et al. (2017) used PNs for safety assessment of unmanned aerial vehicle. A Petri net-based fault modelling approach was proposed in (Sunanda and Seetharamaiah, 2015) and the approach was validated by applying it to a prototype rail-road crossing junction system.

One particular application of Petri nets in system safety and reliability analysis is in the fault detection and diagnosis. Sun et al. (2004) used fuzzy Petri nets to create fault diagnosis models of electric power systems (EPS). By combining Petri nets with fuzzy set theory, this approach allows the fault diagnosis of EPS when incomplete and uncertain alarm information of protective relays and circuit breakers is detected. Feasibility and effectiveness of this approach were illustrated through simulation. Fuzzy PNs have been utilised to propose another approach in (Zhang et al., 2016) for fault diagnosis of power systems. This approach can take into account the temporal behaviour of the systems. Liu et al. (2013) combined fuzzy evidential reasoning (FER) and dynamic adaptive fuzzy Petri nets (DAFPNs) for fault diagnosis and cause analysis. In the approach, FER was used to identify abnormal events and the DAFPNs was used to determine the root causes and consequences of abnormal events. PNs have been used by Mansour et al. (2013) for fault diagnosis of large power generation station. To improve the confidence degree on the diagnostic result, Cheng et al. (2015) proposed a method based on fuzzy PNs.

Case-based reasoning (CBR) (Aamodt and Plaza, 1994) has been integrated with PN by Yang et al. (2004) to propose a method for fault diagnosis of induction motors. In this approach, CBS is used to collect information from previous cases by taking into account knowledge and experience of experts, and thus using this information to solve new problems that show similarity with previous cases. A modular and hierarchical approach has been proposed in (Miyagi and Riascos, 2006) for fault detection and treatment in manufacturing systems. A state-based approach has been proposed by Lefebvre (2012) using the properties of the reachability graph of PNs for fault detection and diagnosis of systems under partial observation of system states and events. Other applications of PNs for fault diagnosis include the fault diagnosis of normal 
and wind turbine generators (Kachur and Shakhova, 2016; Han, 2015).

Basile et al. (2009) proposed a diagnoser for discrete event system (DES), where the online computation of the set of possible fault events explaining the last observed event is done by defining and solving integer linear programming problems. When the system model evolves the diagnoser has to be redesigned and redefined. However, to avoid this, Dotoli et al. $(2008,2009)$ proposed an on-line diagnoser based on Petri nets. Cabasino et al. $(2009,2010)$ also proposed a method for online diagnosis of DES using Petri nets. In this method, faults in the system are modelled as unobservable transitions, and nominal behaviours may also be modelled as unobservable transitions. Renganathan and Bhaskar (2010) also proposed a PN-based method for online fault diagnosis. They have also proposed a method in (Renganathan and Bhaskar, 2013) using hybrid PNs for fault diagnosis and fault tolerant control in bottling plants.

State space explosion is a common problem faced by the PN-based approaches due to the generation of all possible system states. Giua and Seatzu (2005) proposed a method to avoid the exhaustive enumeration of the DES states by introducing the concept of basis markings and justifications. Jiroveanu et al. (2008) proposed a similar approach. This approach made improvement over (Giua and Seatzu, 2005) in the sense that it can be applied to large systems. For fault detection and diagnosis of large scale systems, Jiroveanu and Boel (2006) proposed a model-based approach using timed PNs. Small PN models are created for local components and these models are composed together to obtain the PN model of the whole system. Due to the modularity of the approach, the re-usability of the existing models has increased significantly. This has also improved the scalability and computational complexity of the approach, which allows possible online applications.

\subsubsection{Applications as model-to-model transformation approaches}

An overview of the approaches where different models are translated into PNs for safety and reliability analysis are shown in Table 3. Early application of PNs as model-to-model transformation approaches for system safety and reliability analysis can be found in (Hura and Atwood, 1988) where Hura and Atwood showed the concept of utilisation of PNs for fault tree analysis. They provided an algorithm for mapping classical fault trees into PNs and showed how the PN model can be used for fault detection, fault propagation and reliability analysis. Later on, different complementary contributions had been made showing how PNs can be used to quantify fault trees. For example, Malhotra and Trivedi (1995) developed a methodology for dependability modelling using GSPN and stochastic reward nets (SRN). In their method, algorithms were proposed to convert FTs into equivalent GSPN and SRN by considering repairability of systems. At the same time, Liu and Chiou (1997) have presented a novel method for failure analysis using Petri nets. In addition to showing how fault trees can be converted to PN models, this method also showed how to obtain minimal cut sets and path sets from a PN model. To take into account the repair activities in fault tree analysis, Bobbio et al. (2003a) proposed to convert FTs into stochastic Petri nets. A list of many other similar approaches is shown in Table 3 .

In addition to the classical fault trees, dynamic fault trees have also been translated to PNs for safety and reliability analysis. For instance, Codetta-Raiteri (2005) and Zhang et al. (2009) proposed methodologies to translate DFTs into GSPNs for reliability analysis of dynamic systems. On the other hand, Kabir et al. (2018a, 2015b) showed how temporal fault trees can be translated into GSPNs. Fig. 6 shows the graphical symbols of the commonly used DFT gates and a detailed information about the definitions and functional behaviour of these gates can be found in (Dugan et al., 1992; Kabir et al., 2016). The AND and OR gates are two Boolean gates. The output of an AND gate will become true when all its inputs become true, whereas the OR gate will become true when at least one of its input becomes true. The PAND gate can model sequencing of events and the outcome of this gate will become true if and only if all its input becomes true in left-to-right sequential order. The FDEP gate helps to model a scenario when the operations of some system components are dependent on the operation of another component of the system. In the FDEP gate there is only one trigger event, but there could be multiple functionally dependent events. The occurrence of the trigger event would force the dependent events to occur; by contrast, the occurrence of a dependent event would affect neither the trigger event nor the other dependent events. In a SPARE gate, the left most input is the primary input and other inputs act as secondary inputs. This gate designs a scenario where the spare components are activated in a sequence, i.e., if there are two spare components, then the first spare 
will be activated in case the primary fails; if the first spare fails then the second one will be activated. The outcome of the SPARE gate becomes true if all the input events are true. The SPARE gate could model three types of spares: cold spares, warm spares, and hot spares (Kabir et al., 2016). Sequence-Enforcing gate (SEQ) imposes a sequence on its events such that they must occur in that order. Fig. 7 shows the GSPN models of these logic gates used in classical and dynamic fault trees. A detail description of how the logic gates are translated into GSPN models can be found in (Kabir et al., 2018b). Given these GSPN models of the logic gates, Fig. 8 shows a pseudocode of a function that converts a DFT to GSPN in the course of a depth first traversal of the DFT.

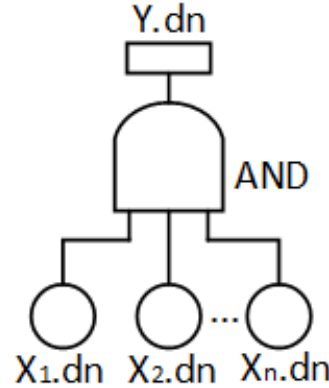

(a)

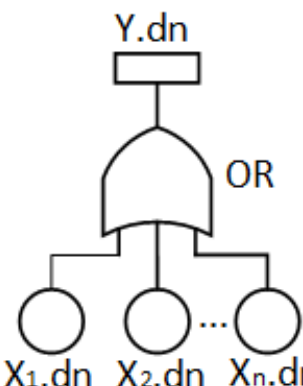

(b)

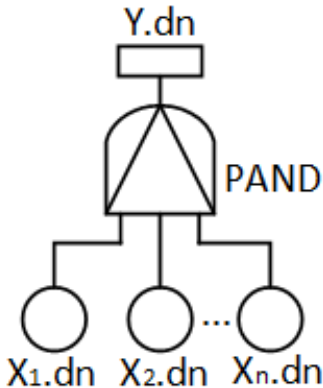

(c)

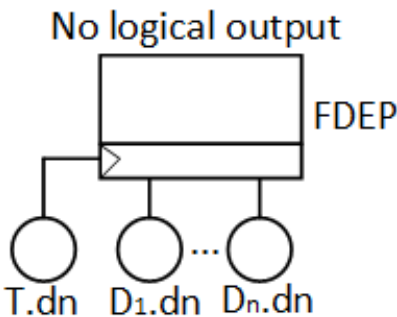

(d)

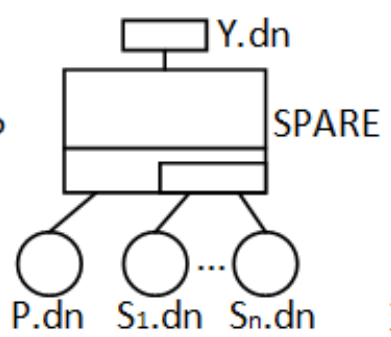

(e)

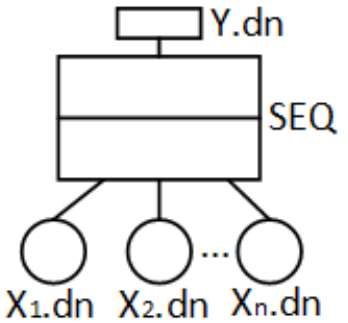

(f)

Figure 6: Commonly used logic gates in DFTs

Table 3: PNs applications as model-to-model transformation approaches

\begin{tabular}{|l|l|}
\hline \multicolumn{1}{|c|}{$\begin{array}{c}\text { Models } \\
\text { translated }\end{array}$} & \multicolumn{1}{c|}{ Contributions } \\
\hline $\begin{array}{l}\text { Classical } \\
\text { fault trees }\end{array}$ & $\begin{array}{l}\text { Hura and Atwood (1988), Malhotra and Trivedi (1995), Liu and Chiou (1997), } \\
\text { Reza et al. (2009), Adamyan and He (2002b) }\end{array}$ \\
\hline $\begin{array}{l}\text { Non-classical } \\
\text { fault trees }\end{array}$ & $\begin{array}{l}\text { Bobbio et al. (2003b), Codetta-Raiteri (2005), Zhang et al. (2009), Kabir et al. (2015b), } \\
\text { Herscheid and Tröger (2014) }\end{array}$ \\
\hline $\begin{array}{l}\text { Reliability } \\
\text { block diagram }\end{array}$ & Robidoux et al. (2010), Signoret et al. (2013) \\
\hline Event Tree & Nỳvlt and Rausand (2012) \\
\hline UML & Hei et al. (2011), Wang and Lu (2012) \\
\hline
\end{tabular}

Petri nets have also been used together with FTA for uncertainty quantification during reliability analysis and a review of such approaches was presented in (Kabir and Papadopoulos, 2018). Knezevic and Odoom (2001) proposed a method for reliability analysis of repairable systems based on Petri nets and fuzzy set 


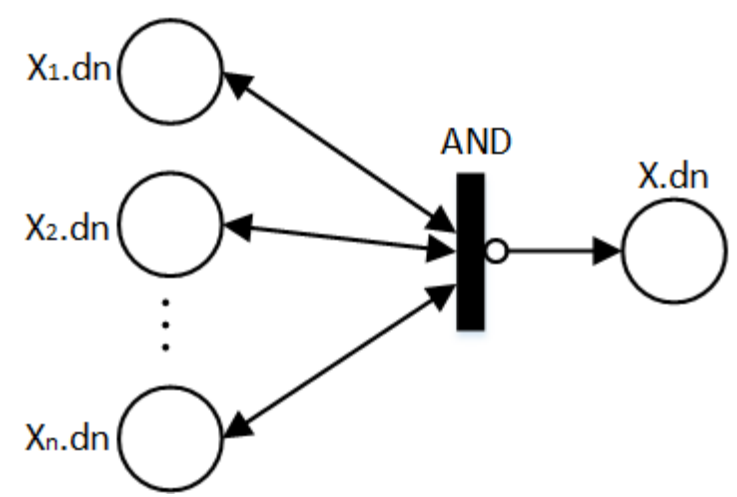

(a) GSPN of AND gate

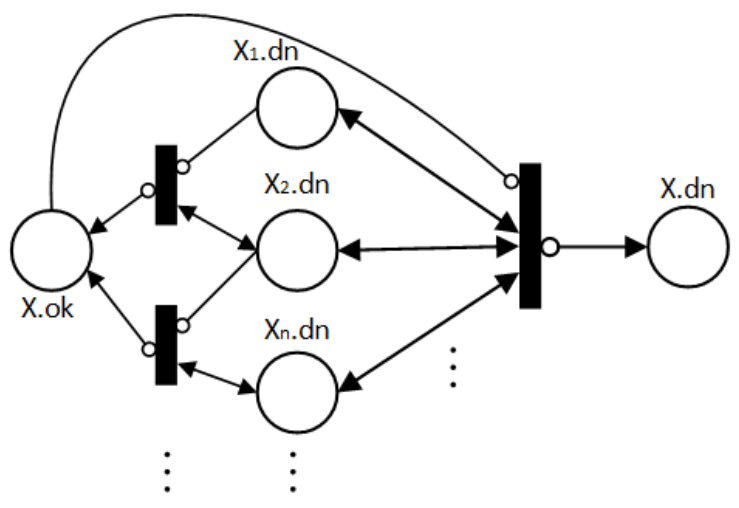

(c) GSPN of PAND gate

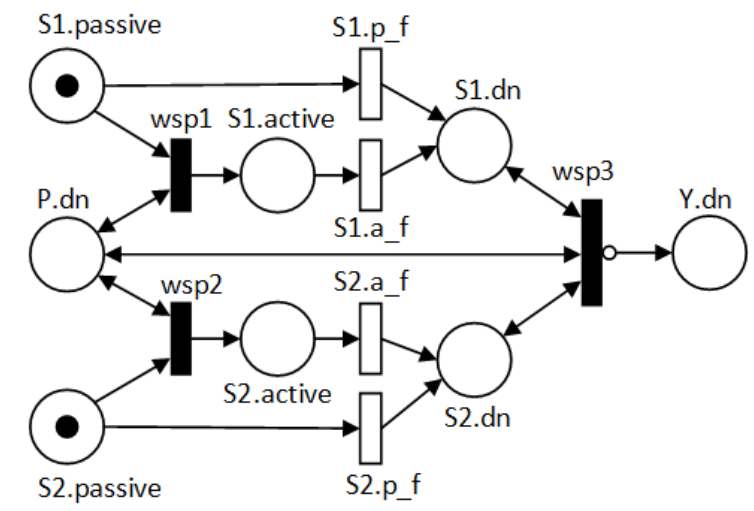

(e) GSPN of SPARE gate

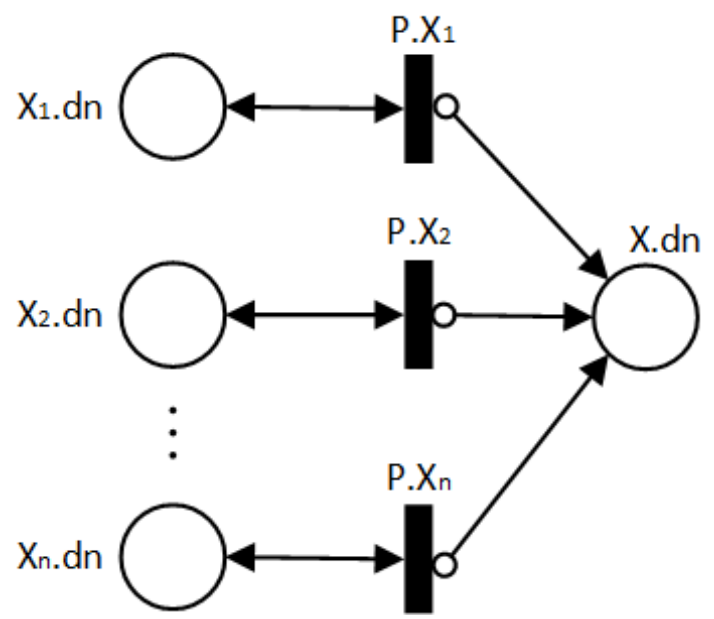

(b) GSPN of OR gate

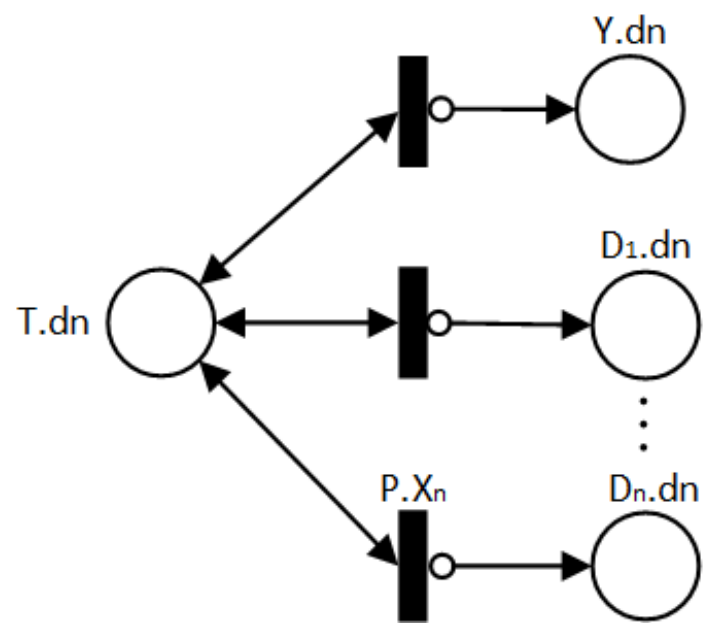

(d) GSPN of FDEP gate

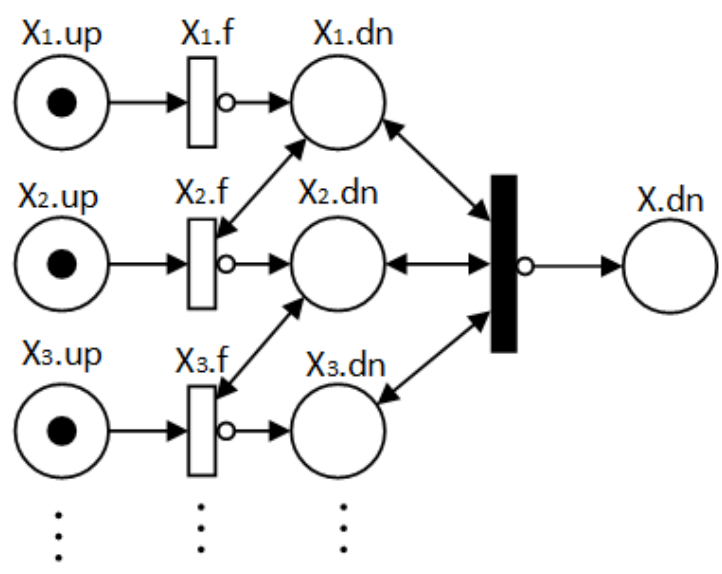

(f) GSPN of SEQ gate

Figure 7: GSPN models of different logic gates 


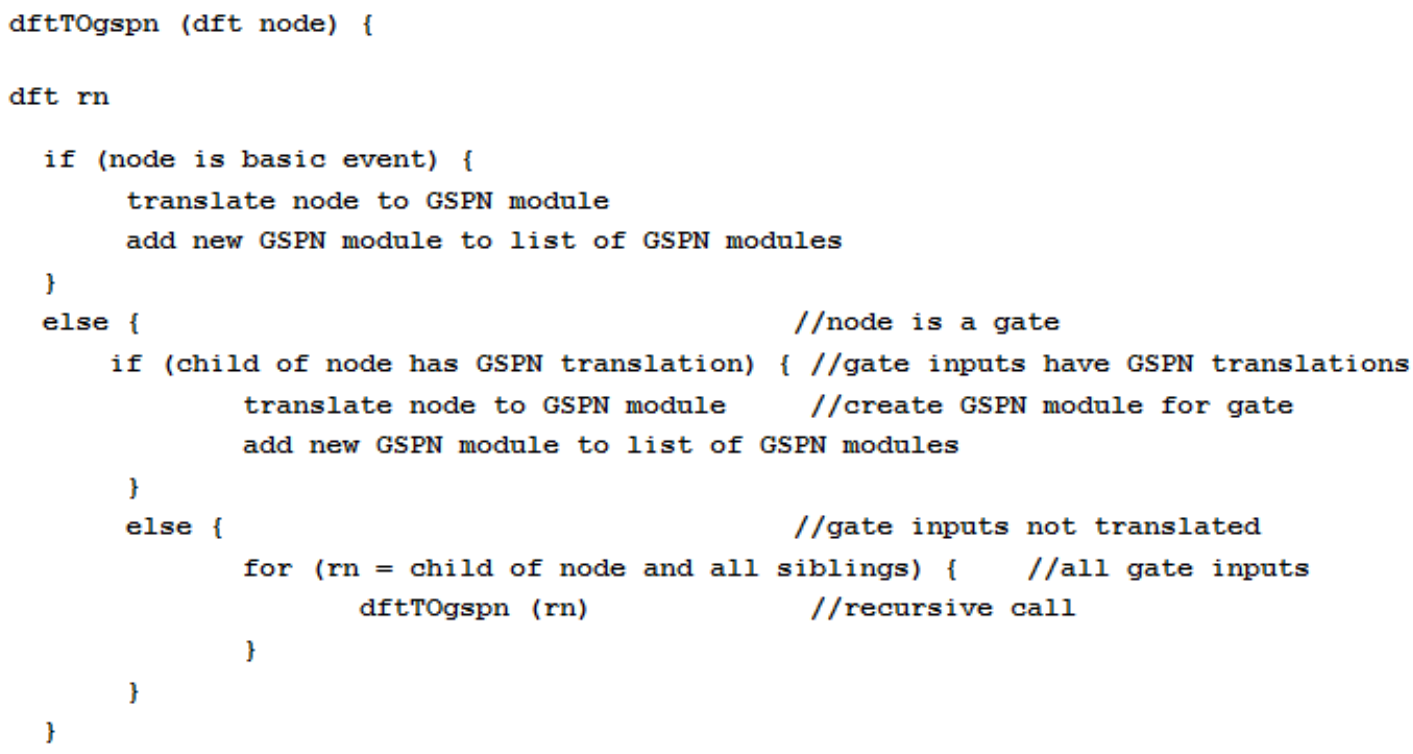

Figure 8: Pseudocode to convert DFT to GSPN (Kabir et al., 2018b)

theory. Fault trees are translated into Petri nets and minimal cut and path sets are generated from PN models. On the other hand, fuzzy set theory has been used for representing failure and repair rates instead of crisp values. Inclusion of fuzzy set in the analysis allowed to incorporate expert opinions, linguistic variables, and uncertainty and imprecision in reliability data. Wu et al. (2011) proposed a method combining FTA and fuzzy reasoning PN (FRPN) for reliability analysis of a solar array system. The root causes of the failure of solar array were determined using FTA. The FT was then translated into FRPN, which was used for fault propagation studies and reliability analysis. This method was extended in (Wu et al., 2012) and applied for reliability optimisation of the solar array of a spacecraft. A PN-based FTA has been used to probabilistic safety assessment of a power plant in (Lee and $\mathrm{Lu}, 2012$ ).

Reliability block diagrams (RBDs) have also been translated to Petri nets for system reliability evaluation. Distefano and Puliafito (2009) have used dynamic RBDs (DRBDs) to capture time-dependant behaviour of systems. DRBDs were translated into PN models for reliability evaluation. Similarly, Robidoux et al. (2010) proposed a method to translate DRBD into colored Petri net for reliability evaluation. A reliability markup language (RML) is defined to formally describe DRBDs. RML is an XML-based language to formally describe the components, structure, and dynamic behaviour of a DRBD model. An algorithm was developed for automatically converting DRBD models to colored Petri net models.

Other models were also translated into PNs for system safety and reliability analysis. For instance, event trees were translated into PNs in (Nỳvlt and Rausand, 2012). Hei et al. (2011) and Wang and Lu (2012) proposed methodologies to translate UML Statecharts into PNs for safety analysis. Dependability artefacts such as fault trees, FMEAs are not only translated to PNs for safety and reliability analysis, they are also used in association with PNs. For example, Dutuit et al. (2008) studied the safety integrity level allocation problem for safety instrumented systems using both FTA and PNs, and compared the results. Fault Tree Driven Markov Processes (FTDMP) (Cacheux et al., 2013) and GSPN have been used together in (Talebberrouane et al., 2016) for availability analysis of safety critical systems and the results were compared to show the advantages of GSPN over FTDMP. In (Yan et al., 2017), PNs have been used with FTA and FMEA for mission modelling of automated guided vehicles. Habchi and Barthod (2016) proposed a methodology combining RBD and PNs for reliability assessment of mechatronic systems. 


\subsection{Applications in Risk Assessments}

Like Bayesian networks, Petri nets have also been used for risk assessment in different industries. In 2003, Vernez et al. (2003) discussed the uses and application perspectives of PNs in the risk analysis and accident modelling areas. A timed Petri net based approach has been proposed by Tuncel and Alpan (2010) for risk assessment and real-time control of supply chain (SC) networks. Failure mode effects and criticality analysis (FMECA) is used to identify the disruption factors in the SC. The dynamic and stochastic behaviour of the $\mathrm{SC}$ is modelled using timed PNs and the risk is evaluated through simulating the model. A special version of PN called attack net has been used by McDermott (2001) for penetration testing to assess the security risk of information systems. This approach allows to view graphically how a collection of flaws can combine together to cause system penetration.

A hierarchical approach was developed by Balasubramanian et al. (2002) to construct PN models for risk analysis of liquid ammonia loading operations in process industries. A set of sub-PN models were created for all the components of the system and the failure behaviour of components was incorporated into these models. The sub-PN models of components were combined together and simulated to obtain the failure propagation behaviour of the system. Getting motivated by the approaches proposed in (McDermott, 2001; Zhou et al., 2003; Dahl, 2005), Henry et al. (2009) proposed a method for assessing the risk of computer network operations against Supervisory Control and Data Acquisition (SCADA) systems. This work extended the earlier work of Balasubramanian et al. (2002) and the process model presented in (Balasubramanian et al., 2002) was extended to construct a corresponding SCADA model by including remote manual and automated control in the model of the process components. Later, this approach was more formalised by the same authors in (Henry et al., 2010).

Helmer et al. (2007) proposed a distributed, agent-based method by combining software fault trees (SFT) with colored Petri nets for systematic specification, design, and implementation of an intrusion detection system (IDS) for security risk assessment of computer systems. SFTs are used for defining intrusions and developing a requirement model of the IDS. The SFT models are then used to create CPN models for intruder detection. Aloini et al. (2012) have showed how colored Petri nets can be used for risk assessment of enterprise resource planning by taking into account dependencies (e.g. causal relationships) among different risk factors. SPNs have been used in (Ghazel, 2009) for dynamic behaviour modelling of level crossings considering both road and railway traffic. An SPN model showing global system behaviour was simulated to evaluate collision risk at level crossings.

UML design and Timed Petri net have been used by Bernardi et al. (2011) for timing-failure risk assessment of real-time software systems. UML-based software specification was augmented with MARTE (OMG, 2009) profile annotations to model the non-functional system properties. The UML-based design is then transformed into a timed PN model for timing-failure risk assessment. Zafra-Cabeza et al. (2004) proposed an algorithm for the optimal scheduling of projects with respect to time and cost, where a timed PN was used to represent the project tasks and the project risk assessment plan. Lee et al. (2013) proposed a PN based method by integrating risk identification, analysis and mitigation actions for qualitative risk assessment of distributed manufacturing system. In this approach, Monte Carlo simulation was used for quantitative risk assessment.

A colored PN-based risk assessment method was proposed by Ammar et al. (1997) for risk assessment of functional specification of real-time software systems. This approach was later extended by the same authors in (Ammar et al., 2001). A colored PN-based method called MORM (man-machine occupational risk modelling) has been developed by Vernez et al. (2004) for occupational health and safety risk assessment in industrial processes. This approach overcomes many of the limitations of the classical risk assessment approaches such as FMEA and HAZOP, by taking into account multiple factors such as man-machine functional interactions, flow deviations, and physical failures of machines into a single analysis. Fanti et al. (2015) used modular colored PNs in their model-based decision support system (DSS) for evaluating risk associated with transport of hazardous materials. The DSS contains three components: the data component (DC), the model component (MC) and the user Interface Component (UIC) can address two problems. Firstly, it can evaluate the risk induced on the population by vehicles transporting hazardous materials through highways. Secondly, it can help to select optimal restoration procedures in critical situations such 
as after accidents involving hazardous material transportation vehicles. The MC within the DSS contains the risk assessment module (RAM), the simulation module (SM) and the decision module (DM). Out of these modules, the SM used colored PNs to model the behaviour of highway network, which includes the model of the accident and the restoration procedure after the accident. Colored PNs have also been used by Stephenson (2004) as a modelling and simulation method for risk assessment of information systems. Kadri et al. (2012) proposed a new method using colored PNs for quantitative risk assessment of domino effect on industrial plants caused by heat radiation to process equipment and/or storage vessels.

A comprehensive risk assessment framework based on fuzzy PNs in combination with the analytic hierarchy process (AHP), the entropy method (EM) and the cloud model, has been proposed by Guo et al. (2016) for long-distance oil and gas transportation pipelines. The AHP method together with the EM method and the cloud model help to address the issue related to the uncertain, vague and random characteristics of risk factors of oil and gas pipelines. On the other hand, Zhou et al. (2017) used weighted fuzzy PNs to propose a method for security risk (e.g., threat of terrorist attacks) assessment in the chemical industry. The use of the PNs helps this method to model interrelationships between risk factors and the importance of the risk factors, thus allowing to perform meaningful risk assessment.

\section{Discussion and future outlook}

Meaningful and accurate safety, reliability, and risk assessment play a vital role in the development and safe operation of safety-critical systems. Several classical approaches such as FTA, ETA and FMEA have been utilised for system analysis. Despite their extensive use for system safety, reliability and risk assessment, they do have a number of shortcomings. The major shortcoming is that these approaches often perform analysis under unrealistic assumptions, such as by considering statistical and stochastic independence between events, binary states of system components, and overlooking temporal behaviour. However, these limitations have not gone unnoticed. Alternative approaches have been developed to alleviate the limitations of classical approaches, thus enabling the analysis of practical systems under realistic assumptions.

Bayesian networks and Petri nets are two different approaches that are used either as standalone approaches or in association with other approaches to address many of the limitations of the existing approaches. The two approaches share capabilities, such as enabling predictive analysis of failure behaviour of systems by taking into account statistical, stochastic and temporal dependencies of events. Moreover, both the approaches can consider repairability and multiple states of failure of components during modelling of the system behaviour. However, they do have distinct capabilities, and relatively better performance in different scenarios. For instance, in diagnostic analysis, BN-based approaches can propagate new evidence through the network to obtain new beliefs about the failure probability of the events and update prior beliefs. Unlike PNs, BNs are therefore able to adapt and refine their diagnostic ability over time.

Although extensive research has been performed on BNs and PNs, there exist many challenges in this area. When BNs and PNs are used as standalone approaches, their manual construction requires experts with extensive knowledge about the system so that behaviour can be accurately captured. In BNs, this task is further complicated by the fact that deep knowledge about the system is required to understand dependencies among different variables in a BN model so that conditional probability tables can be created. If the system design evolves over the life-cycle, the BN or PN model of the system has to be adapted to maintain consistency.

The major limitations of BNs application as standalone approaches are that there are limited formal semantic guidelines available for developing BNs for a system and they do not guarantee a coherent model. For this reason, it is possible to create different BN models for the same system which will produce the same result, except that they differ in complexity. This is primarily a problem when the parent-child relationships among nodes are not properly defined, thus producing a complex model instead of a simple one. On the other hand, strong semantics guidelines and mathematical formulation of PNs could help to create coherent models of systems behaviour, but PN models are inherently complex. For this reason, PNs-based approaches often suffer from the state space explosion problem, which undermines their efficacy and applicability for the analysis of large and complex industrial scale systems. Mechanisms have been proposed to minimise the state space explosion problem. But there still remains scope for further research to improve the scalability 
of the PN approaches. For example, modularisation (Gulati and Dugan, 1997; Anand and Somani, 1998; Manian et al., 1998; Huang and Chang, 2007; Chiacchio et al., 2013; Yevkin, 2011) has been used successfully to improve performance of the techniques, an area where further work is possible. In addition to that, when the state space of PN is too wide for the analysis, simulation can be exploited instead of analysis.

When BNs and PNs are used as part of model-to-model transformation approaches, coherent models can be created as these models are transformed from other artefacts such as fault trees. However, the correctness of the BN or PN models and subsequent analyses depends on the correctness of the input models. Moreover, for PN-based approaches, it can be difficult to translate large models into PNs and the translated models can be very difficult to understand due to very complex graphical view. Again, while running analysis on such a big model the users can face the state space explosion problem. On the other hand, BN-based approaches provide more flexibility and create more understandable models. Moreover, BN-based approaches can avoid the state space explosion problem by avoiding the state space generation by exploiting the local dependencies between variables while modelling complex behaviour. Another advantage of $\mathrm{BN}$, with respect to $\mathrm{PN}$, is the possibility to learn the model, in terms of graphs and probabilities, by applying machine learning techniques on available training data (Neapolitan, 2004). However, BN models are more complex than PN in terms of parameter setting: in the $\mathrm{PN}$, we only have to set firing rates of timed transitions, and they are equivalent to the failure rates of the basic events; in the BN instead, we have to set the CPT entries of each variable, considering all the possible value combinations of the variable with its parent variables. If the entries in a CPT are deterministic (e.g. CPT of a logic gate), then these values can be generated automatically.

In order to have a general comparison between PN and BN based approaches when used as model-tomodel transformation approaches, consider the simple dynamic fault tree (DFT) shown in Fig. 9. The BN model and the GSPN model of this DFT is shown in Figs. 10 and 11, respectively. As can be seen, from a graphical point of view, the PN model of the DFT is relatively more complex than the BN model of the DFT. More specifically, while the BN model has 23 nodes, the PN model has 40 nodes. The BN model has 23 arcs; and the PN model has 78 arcs and 34 transitions. In terms of parameter setting, in the PN model, we need to set the firing rates of 13 timed transitions. The BN model of Fig. 10 was created based on the method shown in (Kabir et al., 2018a) and it is a discrete time model. To facilitate the definition of the temporal behaviour of the logic gates in the CPTs, the time was discretised by dividing it into 5 different intervals. As the node G1 represents the top event of the TFT, time is not discretised for this node. For this particular setting, we need to set 17574 values in the probability tables. Out of these values, 17496 values are deterministic, i.e., either 0 or 1 , hence set automatically. 78 probabilistic values for 13 root nodes corresponding to 13 basic events of the DFT were set manually. A transient analysis performed on this GSPN model for 5000 hours mission time generates 174345 states. DFTs of larger systems can be much larger and more complex, which will lead to graphically complex and computationally demanding PN models. In terms of results, the BN-based and the PN-based methods estimated the top event probability of the DFT as 0.0293 and 0.0290 , respectively. This shows that the results given by the two models are coherent.

In terms of modelling of time, PN-based methods use continuous model of time, whereas BNs-based approaches use both discrete and continuous models of time. When the discrete model of time is used, the granularity of time discretisation must be decided as part of the transformation process. The size of the step $(\Delta)$, in other words, the number of discrete intervals used, represents a trade-off between the computing time and the precision of the results. When an approximate result suffices, using a relatively large step size it is possible to obtain results in short time. On the hand, the selection of a smaller discretisation step can make the model of time nearly continuous. As a result, computing time increases, but in return accuracy of the results improve. To eliminate the need for time discretisation, continuous-time BNs use the continuous model of time. Most recently, a generalised continuous-time BN-based approach (CodettaRaiteri and Portinale, 2017) combined strong features of both BNs and PNs to provide added advantages. For instance, both system repairability and multi-state system are considered in this approach. However, due to the consideration of multiple states for system components and PN-based analysis, this approach may suffer from state space explosion. Further research in this area is thus also required. One potential option is to use approximate algorithms instead of exact algorithms to analyse BNs. For instance, for dynamic BN, the approximate Boyen-Koller algorithm (Boyen and Koller, 1998) can be used instead of the exact Junction 


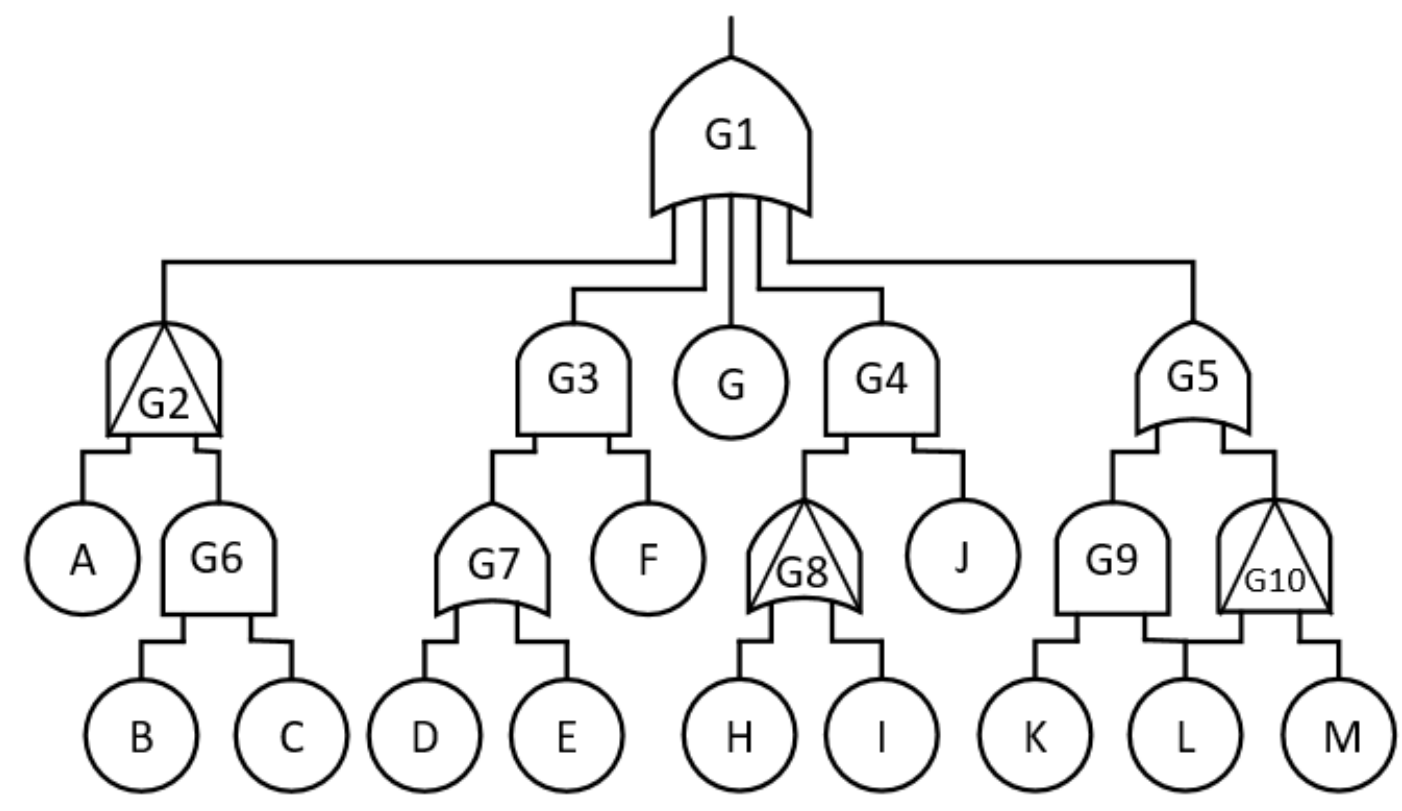

Figure 9: A simple DFT

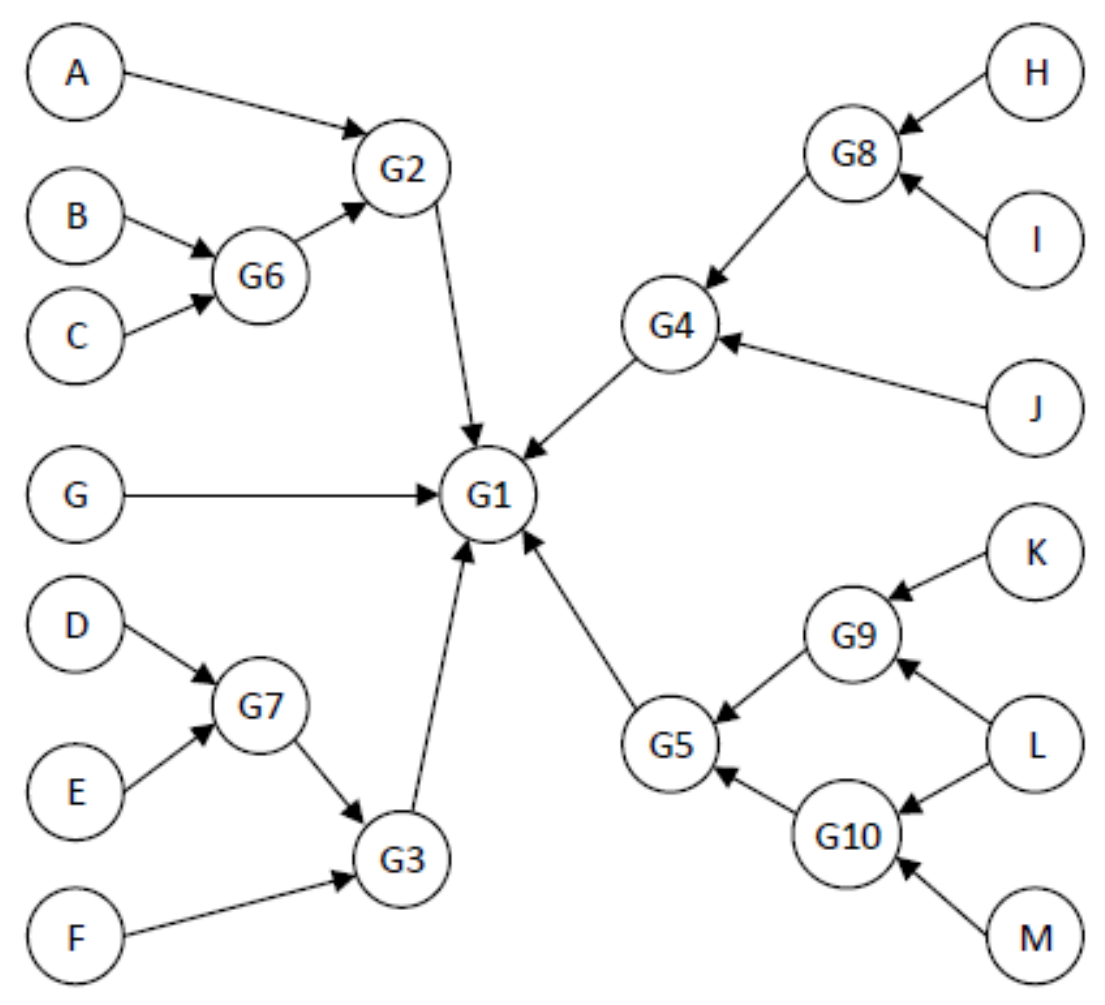

Figure 10: BN model of the DFT of Fig. 9 


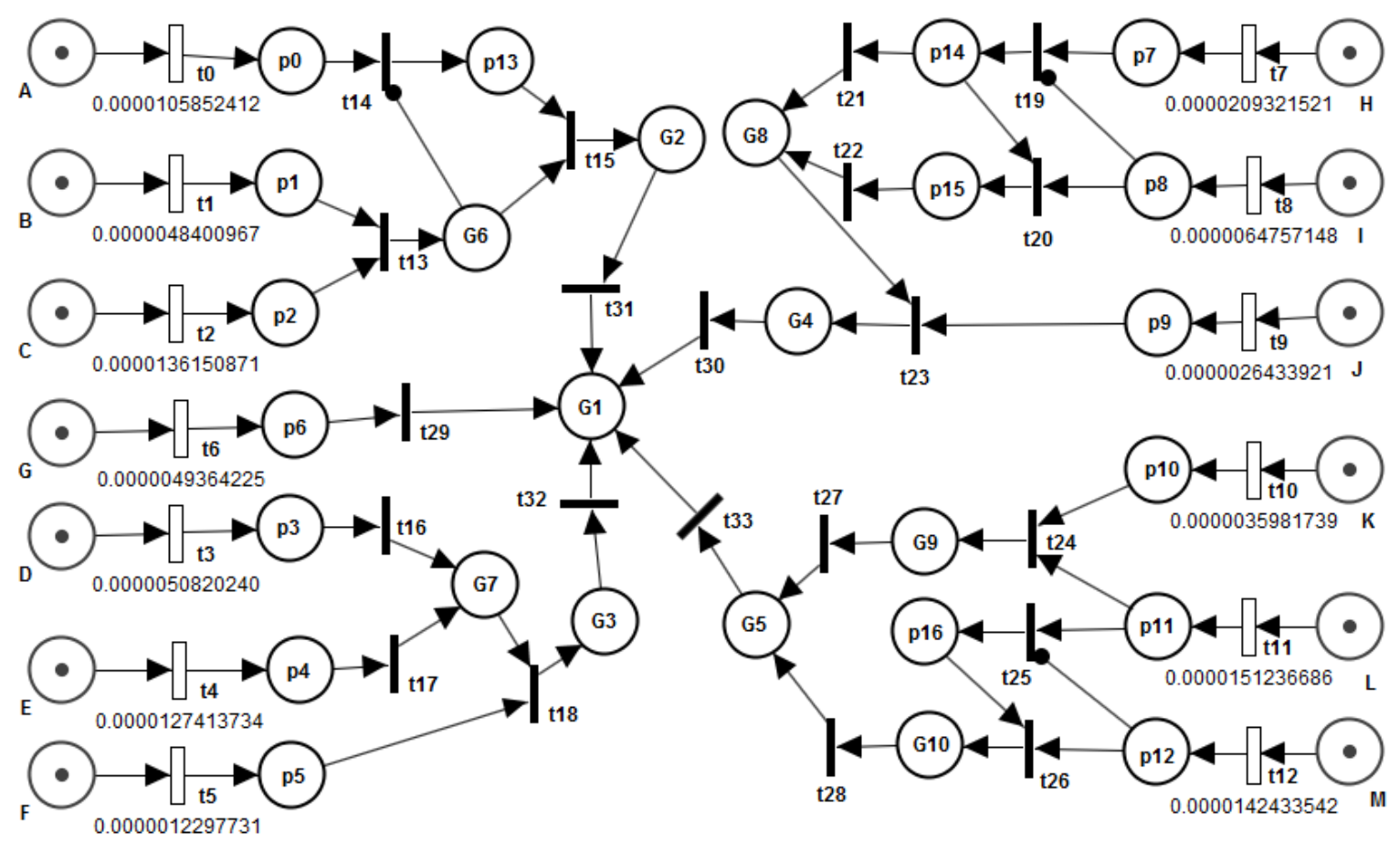

Figure 11: GSPN of the DFT of Fig. 9

Tree algorithm (Murphy, 2002), when the BN is characterized by a relevant complexity.

\section{Conclusion}

Oversimplification of the system analysis processes made by classical approaches based on unrealistic assumptions can lead to an inappropriate estimation of system dependability properties. This shortcoming has necessitated the development of approaches that can perform more accurate analysis by taking into account realistic behaviour of systems. BNs and PNs have been proven effective for system analysis under realistic scenarios and a significant upward trend is noticed in their applications in system safety, reliability, and risk assessment. This paper reviews many such developments and applications.

The popularity of BNs has increased rapidly due to their flexible structure and ability to reasoning under uncertainty. The diagnostic analysis capability of BNs has made them superior techniques for the analysis of practical systems. On the other hand, PNs are particularly suitable for specifying and analysing the behaviour of complex, distributed and concurrent systems. Although PN-based methods are extensively used for performance and performability analysis of systems, their use in safety, reliability, and risk assessment applications is still limited.

While BNs and PNs-based approaches overcome many limitations of the classical safety and reliability analysis approaches, there exist some challenges in this area as discussed in section 5 . Therefore, future research efforts may be directed to address the aforementioned challenges, and thus improve further the application potential of these approaches to a wider range of systems.

\section{Acknowledgements}

This work was funded by the DEIS H2020 project (Grant Agreement 732242). 


\section{References}

\section{References}

Aamodt, A., Plaza, E., 1994. Case-based reasoning: Foundational issues, methodological variations, and system approaches. AI communications 7, 39-59.

Abimbola, M., Khan, F., Khakzad, N., Butt, S., 2015. Safety and risk analysis of managed pressure drilling operation using Bayesian network. Safety science 76, 133-144.

Adamyan, A., He, D., 2002a. Analysis of sequential failures for assessment of reliability and safety of manufacturing systems. Reliability Engineering \& System Safety 76, 227-236.

Adamyan, A., He, D., 2002b. Failure and safety assessment of systems using Petri nets, in: Proceedings of International Conference on Robotics and Automation, IEEE. pp. 1919-1924.

Adamyan, A., He, D., 2003. Sequential failure analysis using counters of Petri net models. IEEE Transactions on Systems, Man, and Cybernetics-part A: Systems and Humans 33, 1-11.

Adamyan, A., He, D., 2004. System failure analysis through counters of Petri net models. Quality and Reliability Engineering International 20, 317-335.

Akhtar, M.J., Utne, I.B., 2014. Human fatigue's effect on the risk of maritime groundings-a bayesian network modeling approach. Safety science $62,427-440$.

Ale, B., Bellamy, L.J., Cooke, R., Goossens, L., Hale, A.R., Roelen, A., Smith, E., 2006. Towards a causal model for air transport safety — an ongoing research project. Safety Science 44, 657-673.

Ale, B.J., Bellamy, L., Van der Boom, R., Cooper, J., Cooke, R.M., Goossens, L.H., Hale, A., Kurowicka, D., Morales, O., Roelen, A., et al., 2009. Further development of a causal model for air transport safety (cats): Building the mathematical heart. Reliability Engineering \& System Safety 94, 1433-1441.

Aljaroudi, A., Khan, F., Akinturk, A., Haddara, M., Thodi, P., 2015. Risk assessment of offshore crude oil pipeline failure. Journal of Loss Prevention in the Process Industries 37, 101-109.

Aloini, D., Dulmin, R., Mininno, V., 2012. Modelling and assessing ERP project risks: A Petri Net approach. European Journal of Operational Research 220, 484-495.

Ammar, H.H., Nikzadeh, T., Dugan, J.B., 1997. A methodology for risk assessment of functional specification of software systems using colored Petri nets, in: Proceedings of Fourth International Software Metrics Symposium, IEEE. pp. $108-117$.

Ammar, H.H., Nikzadeh, T., Dugan, J.B., 2001. Risk assessment of software-system specifications. IEEE transactions on Reliability 50, 171-183.

Anand, A., Somani, A.K., 1998. Hierarchical analysis of fault trees with dependencies, using decomposition, in: Proceedings of the Annual Reliability and Maintainability Symposium, IEEE. pp. 69-75.

Antão, P., Guedes Suares, C., Grande, O., Trucco, P., 2009. Analysis of maritime accident data with BBN models. Safety, reliability and risk analysis: theory, methods and applications. London, UK: Taylor \& Francis Group .

Aslett, L.J., Coolen, F., Wilson, S.P., 2015. Bayesian inference for reliability of systems and networks using the survival signature. Risk Analysis 35, 1640-1651.

Bai, C.G., 2005. Bayesian network based software reliability prediction with an operational profile. Journal of Systems and Software 77, 103-112.

Balakrishnan, M., Trivedi, K.S., 1996. Stochastic petri nets for the reliability analysis of communication network applications with alternate-routing. Reliability Engineering \& System Safety 52, 243-259.

Balasubramanian, N., Chang, C.T., Wang, Y.F., 2002. Petri-net models for risk analysis of hazardous liquid loading operations. Industrial \& engineering chemistry research 41, 4823-4836.

Baraldi, P., Conti, M., Librizzi, M., Zio, E., Podofillini, L., Dang, V., 2009. A bayesian network model for dependence assessment in human reliability analysis, in: Proceedings of the Annual European Safety and Reliability Conference, ESREL, pp. 223230 .

Barua, S., Gao, X., Pasman, H., Mannan, M.S., 2016. Bayesian network based dynamic operational risk assessment. Journal of Loss Prevention in the Process Industries 41, 399-410.

Basile, F., Chiacchio, P., De Tommasi, G., 2009. An efficient approach for online diagnosis of discrete event systems. IEEE Transactions on Automatic Control 54, 748-759.

Bensi, M., Kiureghian, A.D., Straub, D., 2013. Efficient bayesian network modeling of systems. Reliability Engineering \& System Safety 112, 200-213.

Bernardi, S., Campos, J., Merseguer, J., 2011. Timing-failure risk assessment of UML design using Time Petri Net bound techniques. IEEE Transactions on Industrial Informatics 7, 90-104.

Berthomieu, B., Diaz, M., 1991. Modeling and verification of time dependent systems using time Petri nets. IEEE Transactions on Software Engineering 17, 259-273. doi:10.1109/32.75415.

Beyaert, B., Florin, G., Lonc, P., Natkin, S., 1981. Evaluation of computer systems dependability using stochastic Petri nets, in: Digest of the 11th Annual Symposium on Fault-Tolerant Computing, pp. 79-81.

Bhandari, J., Abbassi, R., Garaniya, V., Khan, F., 2015. Risk analysis of deepwater drilling operations using Bayesian network. Journal of Loss Prevention in the Process Industries 38, 11-23.

Bobbio, A., Ciancamerla, E., Franceschinis, G., Gaeta, R., Minichino, M., Portinale, L., 2003a. Sequential application of heterogeneous models for the safetyanalysis of a control system: a case study. Reliability Engineering \& System Safety 81 , 269-280.

Bobbio, A., Franceschinis, G., Gaeta, R., Portinale, L., 1999. Exploiting Petri nets to support fault tree based dependability analysis, in: Petri Nets and Performance Models, 1999. Proceedings. The 8th International Workshop on, IEEE. pp. $146-155$. 
Bobbio, A., Franceschinis, G., Gaeta, R., Portinale, L., 2003b. Parametric fault tree for the dependability analysis of redundant systems and its high-level Petri net semantics. IEEE Transactions on Software Engineering 29, $270-287$.

Bobbio, A., Portinale, L., Minichino, M., Ciancamerla, E., 2001. Improving the analysis of dependable systems by mapping fault trees into Bayesian networks. Reliability Engineering \& System Safety 71, 249-260. doi:10.1016/S0951-8320(00)00077-6.

Boudali, H., Dugan, J.B., 2005a. A discrete-time Bayesian network reliability modeling and analysis framework. Reliability Engineering \& System Safety 87, 337-349.

Boudali, H., Dugan, J.B., 2005b. A new Bayesian network approach to solve dynamic fault trees, in: Proceedings of Annual Reliability and Maintainability Symposium, IEEE. pp. 451-456.

Boudali, H., Dugan, J.B., 2006. A Continuous-Time Bayesian Network Reliability Modeling, and Analysis Framework. IEEE Transaction on Reliability 55, 86-97.

Bouissou, M., Martin, F., Ourghanlian, A., 1999. Assessment of a safety-critical system including software: a Bayesian belief network for evidence sources, in: Reliability and Maintainability Symposium, 1999. Proceedings. Annual, IEEE. pp. 142-150.

Bouissou, M., Pourret, O., 2003. A Bayesian belief network based method for performance evaluation and troubleshooting of multistate systems. International Journal of Reliability, Quality and Safety Engineering 10, 407-416. doi:10.1142/ S0218539303001275.

Boyen, X., Koller, D., 1998. Tractable inference for complex stochastic processes, in: Proceedings of the Fourteenth conference on Uncertainty in artificial intelligence, Morgan Kaufmann Publishers Inc.. pp. 33-42.

Brito, M., Griffiths, G., 2016. A bayesian approach for predicting risk of autonomous underwater vehicle loss during their missions. Reliability Engineering \& System Safety 146, 55-67.

Buchacker, K., 1999. Combining fault trees and Petri nets to model safety-critical systems, in: High performance computing, pp. 439-444.

Cabasino, M.P., Giua, A., Seatzu, C., 2009. Diagnosis of discrete event systems using labeled Petri nets. IFAC Proceedings Volumes $42,52-57$.

Cabasino, M.P., Giua, A., Seatzu, C., 2010. Fault detection for discrete event systems using Petri nets with unobservable transitions. Automatica 46, 1531-1539.

Cacheux, P.J., Collas, S., Dutuit, Y., Folleau, C., Signoret, J.P., Thomas, P., 2013. Assessment of the expected number and frequency of failures of periodically tested systems. Reliability Engineering \& System Safety 118, 61-70.

Cadini, F., Gioletta, A., 2016. A Bayesian Monte Carlo-based algorithm for the estimation of small failure probabilities of systems affected by uncertainties. Reliability Engineering \& System Safety 153, 15-27.

Cai, B., Liu, Y., Liu, Z., Tian, X., Dong, X., Yu, S., 2012. Using bayesian networks in reliability evaluation for subsea blowout preventer control system. Reliability Engineering \& System Safety 108, 32-41.

Cai, B., Liu, Y., Ma, Y., Liu, Z., Zhou, Y., Sun, J., 2015. Real-time reliability evaluation methodology based on dynamic bayesian networks: A case study of a subsea pipe ram bop system. ISA transactions 58, 595-604.

Cai, B., Liu, Y., Zhang, Y., Fan, Q., Liu, Z., Tian, X., 2013. A dynamic bayesian networks modeling of human factors on offshore blowouts. Journal of Loss Prevention in the Process Industries 26, 639-649.

Cavone, G., Dotoli, M., Seatzu, C., 2017. A Survey on Petri Net Models for Freight Logistics and Transportation Systems. IEEE Transactions on Intelligent Transportation Systems .

Cheng, H., He, Z., Wang, Q., Yang, J., Lin, S., 2015. Fault diagnosis method based on petri nets considering service feature of information source devices. Computers \& Electrical Engineering 46, 1-13.

Chengshan, W., Yinghua, X., 2004. Applying Bayesian network to distribution system reliability analysis, in: IEEE Region 10 Conference (TENCON), IEEE. pp. 562-565.

Chiacchio, F., Cacioppo, M., D’Urso, D., Manno, G., Trapani, N., Compagno, L., 2013. A weibull-based compositional approach for hierarchical dynamic fault trees. Reliability Engineering \& System Safety 109, 45-52.

Chin, K.S., Tang, D.W., Yang, J.B., Wong, S.Y., Wang, H., 2009. Assessing new product development project risk by Bayesian network with a systematic probability generation methodology. Expert Systems with Applications 36, 9879-9890.

Chiola, G., Dutheillet, C., Franceschinis, G., Haddad, S., 1991. Stochastic well-formed coloured nets and multiprocessor modelling applications, in: High-level Petri Nets. Springer, pp. 504-530.

Chiola, G., Dutheillet, C., Franceschinis, G., Haddad, S., 1993. Stochastic well-formed colored nets and symmetric modeling applications. IEEE Transactions on Computers 42, 1343-1360.

Chung, L.P., Chang, C.T., 2011. Petri-net models for comprehensive hazard analysis of mocvd processes. Computers \& chemical engineering $35,356-371$.

Codetta-Raiteri, D., 2005. The conversion of dynamic fault trees to stochastic Petri nets, as a case of graph transformation. Electronic Notes in Theoretical Computer Science 127, 45-60.

Codetta-Raiteri, D., 2015. Applying generalized continuous time Bayesian networks to a reliability case study. IFACPapersOnLine 48, 676-681.

Codetta-Raiteri, D., Bobbio, A., 2005. Solving dynamic reliability problems by means of ordinary and fluid stochastic Petri nets, in: Proceedings of the European Safety and Reliability Conference, pp. 381-389.

Codetta-Raiteri, D., Bobbio, A., Montani, S., Portinale, L., 2012. A dynamic bayesian network based framework to evaluate cascading effects in a power grid. Engineering Applications of Artificial Intelligence 25, 683-697.

Codetta-Raiteri, D., Portinale, L., 2010. Generalized continuous time Bayesian networks and their GSPN semantics, in: European Workshop on Probabilistic Graphical Models, pp. 105-112.

Codetta-Raiteri, D., Portinale, L., 2014. Approaching dynamic reliability with predictive and diagnostic purposes by exploiting dynamic Bayesian networks. Proceedings of the Institution of Mechanical Engineers, Part O: Journal of Risk and Reliability 228, 488-503.

Codetta-Raiteri, D., Portinale, L., 2015. Dynamic Bayesian networks for fault detection, identification, and recovery in au- 
tonomous spacecraft. IEEE Transactions on Systems, Man, and Cybernetics: Systems 45, 13-24.

Codetta-Raiteri, D., Portinale, L., 2017. Generalized Continuous Time Bayesian Networks as a modelling and analysis formalism for dependable systems. Reliability Engineering \& System Safety 167, 639-651.

Coolen, F.P., Coolen-Maturi, T., 2013. Generalizing the signature to systems with multiple types of components, in: Complex systems and dependability. Springer, pp. 115-130.

Daemi, T., Ebrahimi, A., Fotuhi-Firuzabad, M., 2012. Constructing the Bayesian network for components reliability importance ranking in composite power systems. International Journal of Electrical Power \& Energy Systems 43, 474-480.

Dahl, O.M., 2005. Using coloured Petri nets in penetration testing. Master's thesis. Gjøvik University College.

Davoudian, K., Wu, J.S., Apostolakis, G., 1994. Incorporating organizational factors into risk assessment through the analysis of work processes. Reliability Engineering \& System Safety 45, 85-105. doi:10.1016/0951-8320(94)90079-5.

Desrochers, A.A., Deal, T.J., Fanti, M.P., 2005. Complex-Valued Token Petri Nets. IEEE Trannsactions on Automation Science and Engineering 2, 309-318.

de Dianous, V., Fiévez, C., 2006. ARAMIS project: A more explicit demonstration of risk control through the use of bow-tie diagrams and the evaluation of safety barrier performance. Journal of Hazardous Materials 130, 220-233. doi:10.1016/j. jhazmat.2005.07.010.

Distefano, S., Puliafito, A., 2009. Reliability and availability analysis of dependent-dynamic systems with DRBDs. Reliability Engineering \& System Safety 94, 1381-1393.

Ditlevsen, O., Madsen, H.O., 1996. Structural reliability methods. volume 178. Wiley New York.

Doguc, O., Ramirez-Marquez, J.E., 2009. A generic method for estimating system reliability using Bayesian networks. Reliability Engineering \& System Safety 94, 542-550.

Doguc, O., Ramirez-Marquez, J.E., 2012. An automated method for estimating reliability of grid systems using Bayesian networks. Reliability Engineering \& System Safety 104, 96-105.

Donat, R., Leray, P., Bouillaut, L., Aknin, P., 2010. A dynamic bayesian network to represent discrete duration models. Neurocomputing 73, 570-577.

Dotoli, M., Fanti, M., Mangini, A., 2008. Fault detection of discrete event systems using Petri nets and integer linear programming. IFAC Proceedings Volumes 41, 6554-6559.

Dotoli, M., Fanti, M.P., Mangini, A.M., Ukovich, W., 2009. On-line fault detection in discrete event systems by Petri nets and integer linear programming. Automatica 45, 2665-2672.

Dugan, J.B., Bavuso, S.J., Boyd, M.A., 1990. Fault Trees and Sequence Dependencies, in: Proceedings of Annual Reliability and Maintainability Symposium, pp. 286-293. doi:10.1109/ARMS.1990.67971.

Dugan, J.B., Bavuso, S.J., Boyd, M.A., 1992. Dynamic fault-tree models for fault-tolerant computer systems. IEEE Transactions on Reliability 41, 363-377. doi:10.1109/24.159800.

Dugan, J.B., Trivedi, K.S., Geist, R.M., Nicola, V.F., 1984. Extended Stochastic Petri Nets: Applications and Analysis. Technical Report. WISCONSIN UNIV-MADISON MOTOR BEHAVIOR LAB.

Dunjó, J., Fthenakis, V., Vílchez, J.A., Arnaldos, J., 2010. Hazard and operability (HAZOP) analysis. A literature review. Journal of Hazardous Materials 173, 19-32. doi:10.1016/j.jhazmat.2009.08.076.

Dutuit, Y., Châtelet, E., Signoret, J.P., Thomas, P., 1997. Dependability modelling and evaluation by using stochastic Petri nets: application to two test cases. Reliability Engineering \& System Safety 55, 117-124.

Dutuit, Y., Innal, F., Rauzy, A., Signoret, J.P., 2008. Probabilistic assessments in relationship with safety integrity levels by using fault trees. Reliability Engineering \& System Safety 93, 1867-1876.

Duval, C., Fallet-Fidry, G., Iung, B., Weber, P., Levrat, E., 2012. A bayesian network-based integrated risk analysis approach for industrial systems: application to heat sink system and prospects development. Proceedings of the Institution of Mechanical Engineers, Part O: Journal of Risk and Reliability 226, 488-507.

Eleye-Datubo, A.G., Wall, A., Wang, J., 2008. Marine and Offshore Safety Assessment by Incorporative Risk Modeling in a Fuzzy-Bayesian Network of an Induced Mass Assignment Paradigm. Risk Analysis 28, 95-112.

Fanti, M.P., Iacobellis, G., Ukovich, W., 2015. A risk assessment framework for hazmat transportation in highways by colored petri nets. IEEE Transactions on Systems, Man, and Cybernetics: Systems 45, 485-495.

Fard, M.J., Ameri, S., Hamadani, A.Z., 2015. Bayesian approach for early stage reliability prediction of evolutionary products, in: Proceedings of the International Conference on Operations Excellence and Service Engineering, pp. 361-371.

Fenton, N., Neil, M., 2012. Risk assessment and decision analysis with Bayesian networks. Crc Press.

Fenton, N., Neil, M., Marquez, D., 2008. Using bayesian networks to predict software defects and reliability. Proceedings of the Institution of Mechanical Engineers, Part O: Journal of Risk and Reliability 222, 701-712.

Fenton, N.E., Neil, M., 2014. Decision support software for probabilistic risk assessment using Bayesian networks. IEEE software .

Filieri, A., Ghezzi, C., Grassi, V., Mirandola, R., 2010. Reliability Analysis of Component-Based Systems with Multiple Failure Modes, in: International Symposium on Component-Based Software Engineering, Springer, Berlin, Heidelberg. pp. 1-20. doi:10.1007/978-3-642-13238-4_1.

Flammini, F., Marrone, S., Mazzocca, N., Vittorini, V., 2006. Modelling system reliability aspects of ERTMS/ETCS by fault trees and Bayesian networks, in: Proc. European Safety and Reliability Conference, ESREL, pp. 2675-2683.

Foulliaron, J., Bouillaut, L., Barros, A., Aknin, P., 2015. Dynamic Bayesian networks for reliability analysis: from a Markovian point of view to semi-markovian approaches. IFAC-PapersOnLine 48, 694-700.

Franke, U., Flores, W.R., Johnson, P., 2009. Enterprise architecture dependency analysis using fault trees and bayesian networks, in: Proceedings of the 2009 Spring Simulation Multiconference, Society for Computer Simulation International. p. 55.

Garg, H., 2013. Reliability analysis of repairable systems using Petri nets and Vague Lambda-Tau methodology. ISA transac- 
tions $52,6-18$.

Geist, R., Trivedi, K., Dugan, J.B., Smotherman, M., 1983. Design of the hybrid automated reliability predictor, in: Proceedings IEEE/AIAA 5th Digital Avionics Systems Conference.

Genrich, H.J., Lautenbach, K., 1981. System modelling with high-level petri nets. Theoretical computer science 13 , $109-135$.

Gertman, D., Blackman, H., Marble, J., Byers, J., Smith, C., 2005. The SPAR-H human reliability analysis method. US Nuclear Regulatory Commission .

Ghahramani, Z., Rasmussen, C.E., 2003. Bayesian monte carlo, in: Advances in neural information processing systems, pp. $505-512$.

Ghasemieh, H., Remke, A., Haverkort, B.R., 2016. Survivability analysis of a sewage treatment facility using hybrid petri nets. Performance evaluation 97, 36-56.

Ghazel, M., 2009. Using stochastic Petri nets for level-crossing collision risk assessment. IEEE transactions on intelligent transportation systems 10, 668-677.

Ghosh, S., Ghosh, D., Mohanta, D.K., 2017. Impact assessment of reliability of phasor measurement unit on situational awareness using generalized stochastic petri nets. International Journal of Electrical Power \& Energy Systems 93, 75-83.

Gilks, W.R., Wild, P., 1992. Adaptive rejection sampling for gibbs sampling. Applied Statistics , 337-348.

Giua, A., Seatzu, C., 2005. Fault detection for discrete event systems using petri nets with unobservable transitions, in: 44th IEEE Conference on Decision and Control and European Control Conference, IEEE. pp. 6323-6328.

Goerlandt, F., Montewka, J., 2014. A probabilistic model for accidental cargo oil outflow from product tankers in a ship-ship collision. Marine pollution bulletin 79, 130-144.

Gokhale, S.S., Wong, W.E., Trivedi, K.S., Horgan, J., 1998. An analytical approach to architecture-based software reliability prediction, in: Proceedings of IEEE International Computer Performance and Dependability Symposium, IEEE. pp. 13-22.

Gonçalves, P., Sobral, J., Ferreira, L., 2017. Unmanned aerial vehicle safety assessment modelling through petri nets. Reliability Engineering \& System Safety 167, 383-393.

Görkemli, L., Ulusoy, S.K., 2010. Fuzzy Bayesian reliability and availability analysis of production systems. Computers \& Industrial Engineering 59, 690-696.

Gran, B., Helminen, A., 2001. A bayesian belief network for reliability assessment. Computer Safety, Reliability and Security , 35-45.

Gribaudo, M., Bobbio, A., Sereno, M., 2001a. Modeling physical quantities in industrial systems using fluid stochastic petri nets, in: Fifth International Workshop on Performability Modeling of Computer and Communication Systems, pp. 81-85.

Gribaudo, M., Sereno, M., Horváth, A., Bobbio, A., 2001b. Fluid stochastic Petri nets augmented with flush-out arcs: Modelling and analysis. Discrete Event Dynamic Systems 11, 97-117.

Groth, K.M., Mosleh, A., 2012. Deriving causal Bayesian networks from human reliability analysis data: A methodology and example model. Proceedings of the Institution of Mechanical Engineers, Part O: Journal of Risk and Reliability 226, $361-379$.

Groth, K.M., Swiler, L.P., 2013. Bridging the gap between HRA research and HRA practice: A Bayesian network version of SPAR-H. Reliability Engineering \& System Safety 115, 33-42.

Gu, Y.K., Yang, Z.X., 2013. Reliability analysis of multi-state systems based on Bayesian network, in: International Conference on Quality, Reliability, Risk, Maintenance, and Safety Engineering (QR2MSE), IEEE. pp. $332-336$.

Guan, X., He, J., Jha, R., Liu, Y., 2012. An efficient analytical Bayesian method for reliability and system response updating based on Laplace and inverse first-order reliability computations. Reliability Engineering \& System Safety 97, 1-13.

Gulati, R., Dugan, J.B., 1997. A modular approach for analyzing static and dynamic fault trees, in: Proceedings of the Annual Reliability and Maintainability Symposium, IEEE. pp. 57-63.

Guo, Y., Meng, X., Wang, D., Meng, T., Liu, S., He, R., 2016. Comprehensive risk evaluation of long-distance oil and gas transportation pipelines using a fuzzy Petri net model. Journal of Natural Gas Science and Engineering 33, 18-29.

Habchi, G., Barthod, C., 2016. An overall methodology for reliability prediction of mechatronic systems design with industrial application. Reliability Engineering \& System Safety 155, 236-254.

Han, Z., 2015. Fault Diagnosis System of Wind Turbine Generator Based on Petri Net, in: Proceedings of the International Conference on Applied Mechanics, Mechatronics and Intelligent Systems, World Scientific. pp. 311-318.

Hänninen, M., 2014. Bayesian networks for maritime traffic accident prevention: benefits and challenges. Accident Analysis \& Prevention 73, 305-312.

Hänninen, M., Banda, O.A.V., Kujala, P., 2014a. Bayesian network model of maritime safety management. Expert Systems with Applications 41, 7837-7846.

Hänninen, M., Kujala, P., 2012. Influences of variables on ship collision probability in a Bayesian belief network model. Reliability Engineering \& System Safety 102, 27-40.

Hänninen, M., Mazaheri, A., Kujala, P., Montewka, J., Laaksonen, P., Salmiovirta, M., Klang, M., 2014b. Expert elicitation of a navigation service implementation effects on ship groundings and collisions in the gulf of finland. Proceedings of the Institution of Mechanical Engineers, Part O: Journal of Risk and Reliability 228, 19-28.

Hei, X., Chang, L., Ma, W., Gao, J., Xie, G., 2011. Automatic transformation from UML statechart to Petri nets for safety analysis and verification, in: International Conference on Quality, Reliability, Risk, Maintenance, and Safety Engineering (ICQR2MSE), IEEE. pp. 948-951.

Helmer, G., Wong, J., Slagell, M., Honavar, V., Miller, L., Wang, Y., Wang, X., Stakhanova, N., 2007. Software fault tree and coloured petri net-based specification, design and implementation of agent-based intrusion detection systems. International Journal of Information and Computer Security 1, 109-142.

Henry, M.H., Layer, R.M., Snow, K.Z., Zaret, D.R., 2009. Evaluating the risk of cyber attacks on SCADA systems via Petri net analysis with application to hazardous liquid loading operations, in: IEEE Conference on Technologies for Homeland 
Security, IEEE. pp. 607-614.

Henry, M.H., Layer, R.M., Zaret, D.R., 2010. Coupled Petri nets for computer network risk analysis. International Journal of Critical Infrastructure Protection 3, 67-75.

Herscheid, L., Tröger, P., 2014. Specification of dynamic fault tree concepts with stochastic petri nets, in: Eighth International Conference on Software Security and Reliability, IEEE. pp. 177-186.

Honari, B., Donovan, J., Murphy, E., 2009. Using Bayesian networks in reliability evaluation for an (r, s)-out-of- $(\mathrm{m}, \mathrm{n})$ : F distributed communication system. Journal of Statistical Planning and Inference 139, 1756-1765.

Hu, C., Wang, P., Youn, B.D., 2015a. Advances in System Reliability Analysis Under Uncertainty, in: Numerical Methods for Reliability and Safety Assessment. Springer International Publishing, Cham, pp. 271-303. doi:10.1007/978-3-319-07167-1_ 9

Hu, J., Huo, L., Guo, L., Yin, J., Xie, Y., 2008. Reliability assessment of power systems based on element time sequential by Bayesian networks, in: 3rd International Conference on Innovative Computing Information and Control, IEEE. pp. 579-579.

Hu, J., Zhang, L., Cai, Z., Wang, Y., Wang, A., 2015b. Fault propagation behavior study and root cause reasoning with dynamic Bayesian network based framework. Process Safety and Environmental Protection 97, 25-36.

Huang, C.Y., Chang, Y.R., 2007. An improved decomposition scheme for assessing the reliability of embedded systems by using dynamic fault trees. Reliability Engineering \& System Safety 92, 1403-1412.

Huang, H.Z., Zuo, M.J., Sun, Z.Q., 2006. Bayesian reliability analysis for fuzzy lifetime data. Fuzzy Sets and Systems 157, $1674-1686$.

Hura, G.S., Atwood, J.W., 1988. The use of Petri nets to analyze coherent fault trees. IEEE Transactions on reliability 37, $469-474$

Ibrahim, W., Beiu, V., 2011. Using Bayesian networks to accurately calculate the reliability of complementary metal oxide semiconductor gates. IEEE Transactions on Reliability $60,538-549$.

Ionescu, D., Ulmeanu, A.P., Constantinescu, A., Rotaru, I., 2006. Reliability modelling of medium voltage distribution systems of nuclear power plants using generalized stochastic petri nets. Computers \& Mathematics with Applications 51, $285-290$.

Jensen, K., 1981. Coloured petri nets and the invariant-method. Theoretical computer science 14, 317-336.

Jensen, K., 2013. Coloured Petri nets: basic concepts, analysis methods and practical use. volume 1. Springer Science \& Business Media.

Jiang, Y., Zhang, H., Song, X., Jiao, X., Hung, W.N., Gu, M., Sun, J., 2013. Bayesian-network-based reliability analysis of plc systems. IEEE transactions on industrial electronics $60,5325-5336$

Jiroveanu, G., Boel, R.K., 2006. A distributed approach for fault detection and diagnosis based on time Petri nets. Mathematics and Computers in Simulation 70, 287-313.

Jiroveanu, G., Boel, R.K., Bordbar, B., 2008. On-line monitoring of large petri net models under partial observation. Discrete Event Dynamic Systems 18, 323-354.

John, A., Yang, Z., Riahi, R., Wang, J., 2016. A risk assessment approach to improve the resilience of a seaport system using bayesian networks. Ocean Engineering 111, 136-147.

Kabir, G., Tesfamariam, S., Francisque, A., Sadiq, R., 2015a. Evaluating risk of water mains failure using a Bayesian belief network model. European Journal of Operational Research 240, 220-234.

Kabir, S., 2016. Compositional Dependability Analysis of Dynamic Systems with Uncertainty. Ph.D. thesis. University of Hull.

Kabir, S., Papadopoulos, Y., 2018. A review of applications of fuzzy sets to safety and reliability engineering. International Journal of Approximate Reasoning 100, 29-55.

Kabir, S., Papadopoulos, Y., Walker, M., Parker, D., Aizpurua, J.I., Lampe, J., Rüde, E., 2017. A model-based extension to HiP-HOPS for dynamic fault propagation studies, in: International Symposium on Model-Based Safety and Assessment, Springer. pp. 163-178. doi:10.1007/978-3-319-64119-5_11.

Kabir, S., Walker, M., Papadopoulos, Y., 2014. Reliability Analysis of Dynamic Systems by Translating Temporal Fault Trees into Bayesian Networks, in: Ortmeier, F., Rauzy, A. (Eds.), Model-Based Safety and Assessment. Springer International Publishing, Cham. volume 8822 of Lecture Notes in Computer Science, pp. 96-109. doi:10.1007/978-3-319-12214-4.

Kabir, S., Walker, M., Papadopoulos, Y., 2015b. Quantitative evaluation of Pandora Temporal Fault Trees via Petri Nets. IFAC-PapersOnLine 48, 458-463. doi:10.1016/j.ifacol.2015.09.569.

Kabir, S., Walker, M., Papadopoulos, Y., 2018a. Dynamic system safety analysis in HiP-HOPS with Petri Nets and Bayesian Networks. Safety Science 105, 55-70.

Kabir, S., Walker, M., Papadopoulos, Y., Rüde, E., Securius, P., 2016. Fuzzy temporal fault tree analysis of dynamic systems. International Journal of Approximate Reasoning 77, 20-37. doi:10.1016/j.ijar.2016.05.006.

Kabir, S., Yazdi, M., Aizpurua, J.I., Papadopoulos, Y., 2018b. Uncertainty-aware dynamic reliability analysis framework for complex systems. IEEE Access 6, 29499 - 29515. doi:10.1109/ACCESS.2018.2843166.

Kachur, S., Shakhova, N., 2016. Turbine generator status diagnostic system based on Petri nets. Nuclear Energy and Technology $2,81-84$.

Kadri, F., Lallement, P., Chatelet, E., 2012. The Quantitative Risk Assessment of domino eff ect on Industrial Plants Using Colored Stochastic Petri Nets , 1-12

Kalantarnia, M., Khan, F., Hawboldt, K., 2009. Dynamic risk assessment using failure assessment and bayesian theory. Journal of Loss Prevention in the Process Industries 22, 600-606.

Kalantarnia, M., Khan, F., Hawboldt, K., 2010. Modelling of bp texas city refinery accident using dynamic risk assessment approach. Process Safety and Environmental Protection 88, 191-199.

Kanazawa, K., 1992. Reasoning about time and probability. Ph.D. thesis. Brown University.

Kang, C., Zhong, L., Haijun, Z., 2015. Research on probabilistic safety analysis approach of flight control system based on Bayesian network. Procedia Engineering 99, 180-184. 
Kelangath, S., Das, P.K., Quigley, J., Hirdaris, S.E., 2012. Risk analysis of damaged ships-a data-driven Bayesian approach. Ships and Offshore Structures 7, 333-347.

Kelly, D.L., Smith, C.L., 2009. Bayesian inference in probabilistic risk assessment- the current state of the art. Reliability Engineering \& System Safety 94, 628-643.

Khakzad, N., 2015. Application of dynamic Bayesian network to risk analysis of domino effects in chemical infrastructures. Reliability Engineering \& System Safety 138, 263-272.

Khakzad, N., Khan, F., Amyotte, P., 2011. Safety analysis in process facilities: Comparison of fault tree and bayesian network approaches. Reliability Engineering \& System Safety 96, 925-932.

Khakzad, N., Khan, F., Amyotte, P., 2012. Dynamic risk analysis using bow-tie approach. Reliability Engineering \& System Safety 104, 36-44.

Khakzad, N., Khan, F., Amyotte, P., 2013a. Dynamic safety analysis of process systems by mapping bow-tie into Bayesian network. Process Safety and Environmental Protection 91, 46-53.

Khakzad, N., Khan, F., Amyotte, P., 2013b. Quantitative risk analysis of offshore drilling operations: A Bayesian approach. Safety science $57,108-117$.

Khakzad, N., Khan, F., Amyotte, P., 2013c. Risk-based design of process systems using discrete-time Bayesian networks. Reliability Engineering \& System Safety 109, 5-17.

Khakzad, N., Khan, F., Amyotte, P., Cozzani, V., 2013d. Domino effect analysis using Bayesian networks. Risk Analysis 33, 292-306.

Kim, M.C., 2011. Reliability block diagram with general gates and its application to system reliability analysis. Annals of Nuclear Energy 38, 2456-2461.

Kim, M.C., Seong, P.H., 2002. Reliability graph with general gates: an intuitive and practical method for system reliability analysis. Reliability Engineering \& System Safety 78, 239-246.

Kim, M.C., Seong, P.H., Hollnagel, E., 2006. A probabilistic approach for determining the control mode in cream. Reliability Engineering \& System Safety 91, 191-199.

Kleijn, H.C.M., Koutny, M., 2004. Process semantics of general inhibitor nets. Information and Computation 190, $18-69$.

Kleyner, A., Volovoi, V., 2010. Application of petri nets to reliability prediction of occupant safety systems with partial detection and repair. Reliability Engineering \& System Safety 95, 606-613.

Knezevic, J., Odoom, E., 2001. Reliability modelling of repairable systems using petri nets and fuzzy lambda-tau methodology. Reliability Engineering \& System Safety 73, 1-17.

Kumar, V., Aggarwal, K., 1993. Petri net modelling and reliability evaluation of distributed processing systems. Reliability Engineering \& System Safety 41, 167-176.

Labeau, P., Smidts, C., Swaminathan, S., 2000. Dynamic reliability: towards an integrated platform for probabilistic risk assessment. Reliability Engineering \& System Safety 68, 219-254. doi:10.1016/S0951-8320(00)00017-X.

Langseth, H., Portinale, L., 2007. Bayesian networks in reliability. Reliability Engineering and System Safety 92 , 92-108. doi:10.1016/j.ress.2005.11.037.

Lee, A., Lu, L., 2012. Petri net modeling for probabilistic safety assessment and its application in the air lock system of a CANDU nuclear power plant. Procedia Engineering 45, 11-20.

Lee, C., Lv, Y., Hong, Z., 2013. Risk modelling and assessment for distributed manufacturing system. International Journal of Production Research 51, 2652-2666.

Lee, C.J., Lee, K.J., 2006. Application of Bayesian network to the probabilistic risk assessment of nuclear waste disposal. Reliability Engineering \& System Safety 91, 515-532.

Lefebvre, D., 2012. Diagnosis with Petri nets according to partial events and states observation. IFAC Proceedings Volumes 45, 1244-1249.

Leu, S.S., Chang, C.M., 2013. Bayesian-network-based safety risk assessment for steel construction projects. Accident Analysis \& Prevention 54, 122-133.

Leveson, N., Dulac, N., Marais, K., Carroll, J., 2009. Moving Beyond Normal Accidents and High Reliability Organizations: A Systems Approach to Safety in Complex Systems. Organization Studies 30, 227-249. doi:10.1177/0170840608101478.

Leveson, N.G., Stolzy, J.L., 1987. Safety Analysis Using Petr Nets. IEEE Transactions on Software Engineering $13,386-397$.

Li, K., Yi, R., Ma, Z., 2016a. Reliability analysis of dynamic reliability blocks through conversion into dynamic Bayesian networks, in: IEEE International Conference on Industrial Engineering and Engineering Management (IEEM), IEEE. pp. $1330-1334$.

Li, K.X., Yin, J., Bang, H.S., Yang, Z., Wang, J., 2014. Bayesian network with quantitative input for maritime risk analysis. Transportmetrica A: Transport Science 10, 89-118.

Li, L., Wang, J., Leung, H., Jiang, C., 2010. Assessment of catastrophic risk using Bayesian network constructed from domain knowledge and spatial data. Risk Analysis 30, 1157-1175.

Li, P.c., Chen, G.h., Dai, L.c., Zhang, L., 2012. A fuzzy bayesian network approach to improve the quantification of organizational influences in hra frameworks. Safety science 50, 1569-1583.

Li, W., Zuo, M.J., 2008. Reliability evaluation of multi-state weighted k-out-of-n systems. Reliability Engineering \& System Safety 93, 160-167. doi:10.1016/j.ress.2006.11.009.

Li, X., Chen, G., Zhu, H., 2016b. Quantitative risk analysis on leakage failure of submarine oil and gas pipelines using bayesian network. Process Safety and Environmental Protection 103, 163-173.

Li, X., Chen, G., Zhu, H., Zhang, R., 2017. Quantitative risk assessment of submarine pipeline instability. Journal of Loss Prevention in the Process Industries 45, 108-115.

Li, Y., Chen, Y., Tang, N., Yang, L., 2016c. Modeling and analysis of failure mechanism dependence based on petri net, in: Prognostics and System Health Management Conference, IEEE. pp. 1-7. 
Li, Y.F., Mi, J., Liu, Y., Yang, Y.J., Huang, H.Z., 2015. Dynamic fault tree analysis based on continuous-time Bayesian networks under fuzzy numbers. Proceedings of the Institution of Mechanical Engineers, Part O: Journal of Risk and Reliability , 1-12doi:10.1177/1748006X15588446.

Limin, H., Yongli, Z., Gaofeng, F., 2002. Reliability assessment of power systems by bayesian networks, in: Proceedings of International Conference on Power System Technology, IEEE. pp. 876-879.

Lisnianski, A., Levitin, G., 2003. Multi-State System Reliability: Assessment, Optimization and Applications. volume 6 of Series on Quality, Reliability and Engineering Statistics. WORLD SCIENTIFIC. doi:10.1142/5221.

Liu, H.C., Lin, Q.L., Ren, M.L., 2013. Fault diagnosis and cause analysis using fuzzy evidential reasoning approach and dynamic adaptive fuzzy Petri nets. Computers \& Industrial Engineering 66, 899-908.

Liu, K.F.R., Lu, C.F., Chen, C.W., Shen, Y.S., 2012. Applying Bayesian belief networks to health risk assessment. Stochastic environmental research and risk assessment 26, 451-465.

Liu, T., Chiou, S., 1997. The application of Petri nets to failure analysis. Reliability Engineering \& System Safety $57,129-142$.

Liu, Z., Liu, Y., Cai, B., Zhang, D., Zheng, C., 2015. Dynamic Bayesian network modeling of reliability of subsea blowout preventer stack in presence of common cause failures. Journal of Loss Prevention in the Process Industries 38, 58-66.

Lu, J.L., Yang, X.D., Li, R., Zhu, Y.L., 2004. Application of bayesian network in reliability evaluation of power distribution system. Journal of North China Electric Power University 31, 16-19.

Lynn, N., Singpurwalla, N., Smith, A., 1998. Bayesian assessment of network reliability. SIAM review 40, $202-227$.

Maglogiannis, I., Zafiropoulos, E., Platis, A., Lambrinoudakis, C., 2006. Risk analysis of a patient monitoring system using Bayesian network modeling. Journal of Biomedical Informatics 39, 637-647.

Mahadevan, S., Rebba, R., 2005. Validation of reliability computational models using Bayes networks. Reliability Engineering \& System Safety 87, 223-232.

Mahadevan, S., Zhang, R., Smith, N., 2001. Bayesian networks for system reliability reassessment. Structural Safety 23, $231-251$.

Malhotra, M., Trivedi, K.S., 1995. Dependability modeling using Petri-nets. IEEE Transactions on Reliability 44, 428-440.

Manian, R., Dugan, J.B., Coppit, D., Sullivan, K.J., 1998. Combining various solution techniques for dynamic fault tree analysis of computer systems, in: Proceedings of Third IEEE International High-Assurance Systems Engineering Symposium, IEEE. pp. 21-28.

Mansour, M., Wahab, M.A., Soliman, W.M., 2013. Petri nets for fault diagnosis of large power generation station. Ain Shams Engineering Journal 4, 831-842.

Marquez, D., Neil, M., Fenton, N., 2007. A new bayesian network approach to reliability modelling. Mathematical Methods in Reliability (MMR07) .

Marquez, D., Neil, M., Fenton, N., 2008. Solving Dynamic Fault Trees using a New Hybrid Bayesian Network Inference Algorithm, in: 16th Mediterranean Conference on Control and Automation, IEEE. pp. 609-614.

Marquez, D., Neil, M., Fenton, N., 2010. Improved reliability modeling using Bayesian networks and dynamic discretization. Reliability Engineering \& System Safety 95, 412-425.

Marsan, M.A., Balbo, G., Conte, G., Donatelli, S., Franceschinis, G., 1996. Modeling With Generalized Stochastic Petri Nets. Wiley, West Sussex. URL: http://www.di.unito.it/\{ \}greatspn/GSPN-Wiley/.

Marsan, M.A., Chiola, G., 1987. On Petri nets with deterministic and exponentially distributed firing times. Advances in Petri Nets 266, 132-145.

Martins, M.R., Maturana, M.C., 2013. Application of Bayesian Belief networks to the human reliability analysis of an oil tanker operation focusing on collision accidents. Reliability Engineering \& System Safety 110, 89-109.

McDermott, J.P., 2001. Attack net penetration testing, in: Proceedings of the 2000 workshop on New security paradigms, ACM. pp. 15-21.

Mi, J., Li, Y., Huang, H.Z., Liu, Y., Zhang, X., 2012. Reliability analysis of multi-state systems with common cause failure based on Bayesian networks, in: International Conference on Quality, Reliability, Risk, Maintenance, and Safety Engineering (ICQR2MSE), IEEE. pp. 1117-1121.

Mi, J., Li, Y.F., Yang, Y.J., Peng, W., Huang, H.Z., 2016. Reliability assessment of complex electromechanical systems under epistemic uncertainty. Reliability Engineering \& System Safety 152, 1-15.

Miyagi, P., Riascos, L.A.M., 2006. Modeling and analysis of fault-tolerant systems for machining operations based on Petri nets. Control Engineering Practice 14, 397-408.

Mkrtchyan, L., Podofillini, L., Dang, V.N., 2015. Bayesian belief networks for human reliability analysis: A review of applications and gaps. Reliability engineering \& system safety 139, 1-16.

Mkrtchyan, L., Podofillini, L., Dang, V.N., 2016. Methods for building Conditional Probability Tables of Bayesian Belief Networks from limited judgment: An evaluation for Human Reliability Application. Reliability Engineering \& System Safety 151, 93-112.

Molloy, M.K., 1982. Performance analysis using stochastic Petri nets. IEEE Transactions on Computers c-31, 913-917.

Montani, S., Portinale, L., Bobbio, A., 2005. Dynamic Bayesian networks for modeling advanced fault tree features in dependability analysis, in: Proceedings of the sixteenth European conference on safety and reliability, pp. 1415-22.

Montani, S., Portinale, L., Bobbio, A., Codetta-Raiteri, D., 2006a. Automatically translating dynamic fault trees into dynamic Bayesian networks by means of a software tool, in: The First International Conference on Availability, Reliability and Security, IEEE. pp. 1-6.

Montani, S., Portinale, L., Bobbio, A., Codetta-Raiteri, D., 2008. RADYBAN: A tool for reliability analysis of dynamic fault trees through conversion into dynamic Bayesian networks. Reliability Engineering \& System Safety 93, 922-932.

Montani, S., Portinale, L., Bobbio, A., Varesio, M., Codetta-Raiteri, D., 2006b. A tool for automatically translating dynamic fault trees into dynamic Bayesian networks, in: Annual Reliability and Maintainability Symposium, IEEE. pp. 434-441. 
Montewka, J., Ehlers, S., Goerlandt, F., Hinz, T., Tabri, K., Kujala, P., 2014. A framework for risk assessment for maritime transportation systems - a case study for open sea collisions involving RoPax vessels. Reliability Engineering \& System Safety $124,142-157$.

Muppala, J.K., Lin, C., 1996. Dependability analysis of large-scale distributed systems using stochastic petri nets, in: IEEE International Conference on Systems, Man, and Cybernetics, IEEE. pp. 3033-3038.

Mura, I., Bondavalli, A., 2001. Markov regenerative stochastic Petri nets to model and evaluate phased mission systems dependability. IEEE Transactions on Computers 50, 1337-1351.

Murata, T., 1989. Petri nets: Properties, analysis and applications. Proceedings of the IEEE 77, 541-580.

Murphy, K.P., 2002. Dynamic Bayesian Networks: Representation, Inference and Learning. Ph.D. thesis. University of California, Berkeley.

Musharraf, M., Bradbury-Squires, D., Khan, F., Veitch, B., MacKinnon, S., Imtiaz, S., 2014. A virtual experimental technique for data collection for a Bayesian network approach to human reliability analysis. Reliability Engineering \& System Safety $132,1-8$.

Musharraf, M., Hassan, J., Khan, F., Veitch, B., MacKinnon, S., Imtiaz, S., 2013. Human reliability assessment during offshore emergency conditions. Safety science 59, 19-27.

Nannapaneni, S., Mahadevan, S., 2016. Reliability analysis under epistemic uncertainty. Reliability Engineering \& System Safety 155, 9-20. doi:10.1016/j.ress.2016.06.005.

Neapolitan, R.E., 2004. Learning Bayesian networks. volume 38. Pearson Prentice Hall Upper Saddle River, NJ.

Neil, M., Fenton, N., Forey, S., Harris, R., 2001. Using Bayesian belief networks to predict the reliability of military vehicles. Computing \& Control Engineering Journal 12, 11-20.

Neil, M., Littlewood, B., Fenton, N., 1996. Applying bayesian belief networks to system dependability assessment, in: SafetyCritical Systems: The Convergence of High Tech and Human Factors. Springer, pp. 71-94.

Neil, M., Marquez, D., 2012. Availability modelling of repairable systems using bayesian networks. Engineering Applications of Artificial Intelligence 25, 698-704

Neil, M., Tailor, M., Marquez, D., Fenton, N., Hearty, P., 2008. Modelling dependable systems using hybrid Bayesian networks. Reliability Engineering \& System Safety 93, 933-939.

Németh, E., Bartha, T., Fazekas, C., Hangos, K.M., 2009. Verification of a primary-to-secondary leaking safety procedure in a nuclear power plant using coloured petri nets. Reliability Engineering \& System Safety 94, $942-953$.

Nicholson, A.E., 1992. Monitoring discrete environments using dynamic belief networks. Ph.D. thesis. University of Oxford.

Nodelman, U., Shelton, C.R., Koller, D., 2002. Continuous time Bayesian networks, in: Proceedings of the Eighteenth conference on Uncertainty in artificial intelligence, Morgan Kaufmann Publishers Inc.. pp. 378-387.

Nỳvlt, O., Rausand, M., 2012. Dependencies in event trees analyzed by Petri nets. Reliability Engineering \& System Safety $104,45-57$.

OMG, 2009. A UML Profile for Modeling and Analysis of Real Time Embedded Systems (MARTE), document ptc/09-11-02. Technical Report.

Palshikar, G.K., 2002. Temporal fault trees. Information and Software Technology 44, 137-150.

Pearl, J., 1988. Probabilistic reasoning in intelligent systems: Networks of Plausible Inference. Morgan Kaufmann, San Francisco, California.

Peterson, J.L., 1977. Petri nets. ACM Computing Surveys (CSUR) 9, 223-252.

Peterson, J.L., 1980. A note on colored petri nets. Information processing letters 11, 40-43.

Peterson, J.L., 1981. Petri Net Theory and the Modeling of Systems. Prentice Hall PTR, Upper Saddle River, NJ, USA.

Pham, H., 1999. Reliability analysis for dynamic configurations of systems with three failure modes. Reliability Engineering \& System Safety 63, 13-23. doi:10.1016/S0951-8320(98)00006-4.

Pham, H., 2003. Reliability of Systems with Multiple Failure Modes, in: Handbook of Reliability Engineering. Springer-Verlag, London, pp. 19-36. doi:10.1007/1-85233-841-5_2.

Pinna, B., Babykina, G., Brinzei, N., Pétin, J.F., 2013. Using coloured petri nets for integrated reliability and safety evaluations. IFAC Proceedings Volumes 46, 19-24.

Podofillini, L., Dang, V.N., 2013. A bayesian approach to treat expert-elicited probabilities in human reliability analysis model construction. Reliability Engineering \& System Safety 117, 52-64.

Portinale, L., Bobbio, A., 1999. Bayesian networks for dependability analysis: an application to digital control reliability, in: Proceedings of the fifteenth conference on uncertainty in artificial intelligence, Morgan Kaufmann Publishers Inc.. pp. $551-558$.

Portinale, L., Codetta-Raiteri, D., Montani, S., 2010. Supporting reliability engineers in exploiting the power of dynamic Bayesian networks. International journal of approximate reasoning 51, 179-195.

Portugal, P., Carvalho, A., Vasques, F., 2005. A model based on a stochastic petri net approach for dependability evaluation of controller area networks. IFAC Proceedings Volumes 38, 150-157.

Rathnayaka, S., Khan, F., Amyotte, P., 2011. SHIPP methodology: predictive accident modeling approach. Part II. Validation with case study. Process safety and environmental protection 89, 75-88.

Reer, B., Dang, V.N., 2007. The commission errors search and assessment (CESA) method. Paul Scherrer Institute.

Reer, B., Dang, V.N., Hirschberg, S., 2004. The cesa method and its application in a plant-specific pilot study on errors of commission. Reliability Engineering \& System Safety 83, 187-205.

Reisig, W., 2012. Petri nets: an introduction. volume 4. Springer Science \& Business Media.

Ren, J., Jenkinson, I., Wang, J., Xu, D., Yang, J., 2009. An offshore risk analysis method using fuzzy bayesian network. Journal of Offshore Mechanics and Arctic Engineering 131, 041101.

Renganathan, K., Bhaskar, V., 2010. Observer based on-line fault diagnosis of continuous systems modeled as Petri nets. ISA 
transactions 49, 587-595.

Renganathan, K., Bhaskar, V., 2013. Modeling, analysis and performance evaluation for fault diagnosis and Fault Tolerant Control in bottle-filling plant modeled using Hybrid Petri nets. Applied Mathematical Modelling 37, $4842-4859$.

Reza, H., Pimple, M., Krishna, V., Hildle, J., 2009. A safety analysis method using Fault Tree analysis and Petri Nets, in: Sixth International Conference on Information Technology: New Generations, IEEE. pp. 1089-1094.

Robidoux, R., Xu, H., Xing, L., Zhou, M., 2010. Automated modeling of dynamic reliability block diagrams using colored Petri nets. IEEE Transactions on Systems, Man, and Cybernetics-Part A: Systems and Humans 40, 337-351.

Roshandel, R., Medvidovic, N., Golubchik, L., 2007. A bayesian model for predicting reliability of software systems at the architectural level. Software architectures, components, and applications , 108-126.

Sachdeva, A., Kumar, D., Kumar, P., 2008. Reliability analysis of pulping system using petri nets. International Journal of Quality \& Reliability Management 25, 860-877.

Sadou, N., Demmou, H., 2009. Reliability analysis of discrete event dynamic systems with petri nets. Reliability Engineering \& System Safety 94, 1848-1861.

Salem, A.B., Muller, A., Weber, P., 2006. Dynamic Bayesian networks in system reliability analysis. IFAC Proceedings Volumes $39,444-449$

Schneeweiss, W.G., 2001. Tutorial: Petri nets as a graphical description medium for many reliability scenarios. IEEE Transactions on Reliability 50, 159-164.

Seong, P.H. (Ed.), 2008. Reliability and risk issues in large scale safety-critical digital control systems. Springer Science \& Business Media.

Shafer, G., 1976. A mathematical theory of evidence. volume 1. Princeton university press Princeton.

Signoret, J.P., 2009. Dependability \& safety modeling and calculation: Petri nets. IFAC Proceedings Volumes 42, 203-208

Signoret, J.P., Dutuit, Y., Cacheux, P.J., Folleau, C., Collas, S., Thomas, P., 2013. Make your petri nets understandable: Reliability block diagrams driven petri nets. Reliability Engineering \& System Safety 113, 61-75.

Sigurdsson, J., Walls, L., Quigley, J., 2001. Bayesian belief nets for managing expert judgement and modelling reliability. Quality and Reliability Engineering International 17, 181-190.

Simon, C., Weber, P., Evsukoff, A., 2008. Bayesian networks inference algorithm to implement Dempster Shafer theory in reliability analysis. Reliability Engineering \& System Safety 93, 950-963.

Simon, C., Weber, P., Levrat, E., 2007. Bayesian networks and evidence theory to model complex systems reliability. Journal of Computers (JCP) 2, 33-43.

Singh, H., Cortellessa, V., Cukic, B., Gunel, E., Bharadwaj, V., 2001. A bayesian approach to reliability prediction and assessment of component based systems, in: Proceedings of 12th International Symposium on Software Reliability Engineering (ISSRE), IEEE. pp. 12-21.

Singh, K., Raj, N., Sahu, S., Behera, R., Sarkar, S., Maiti, J., 2017. Modelling safety of gantry crane operations using Petri nets. International journal of injury control and safety promotion 24, 32-43.

Singh, L.K., Rajput, H., 2016. Ensuring safety in design of safety critical computer based systems. Annals of Nuclear Energy 92, 289-294.

Singh, L.K., Rajput, H., 2017. Dependability Analysis of Safety Critical Real-Time Systems by Using Petri Nets. IEEE Transactions on Control Systems Technology , 1-17.

Stephenson, P.R., 2004. A formal model for information risk analysis using colored Petri nets, in: Proceedings of the Fifth Workshop and Tutorial on Practical Use of Coloured Petri Nets and the CPN Tools, pp. 167-184.

Straub, D., Kiureghian, A.D., 2010. Bayesian network enhanced with structural reliability methods: methodology. Journal of engineering mechanics 136, 1248-1258.

Straub, D., Papaioannou, I., 2014. Bayesian updating with structural reliability methods. Journal of Engineering Mechanics $141,04014134$.

Su, A., Fan, M., Li, Z., 2012. The reliability analysis of distribution system based on dynamic Bayesian network, in: China International Conference on Electricity Distribution (CICED), IEEE. pp. 1-4.

$\mathrm{Su}, \mathrm{H}$., Che, Y., 2013. Reliability assessment of CTCS-3 using Bayesian networks, in: International Conference on Quality, Reliability, Risk, Maintenance, and Safety Engineering (QR2MSE), IEEE. pp. 284-288.

$\mathrm{Su}$, X., Mahadevan, S., Xu, P., Deng, Y., 2014. Inclusion of task dependence in human reliability analysis. Reliability Engineering \& System Safety 128, 41-55.

Sun, J., Qin, S.Y., Song, Y.H., 2004. Fault diagnosis of electric power systems based on fuzzy Petri nets. IEEE Transactions on Power Systems 19, 2053-2059.

Sunanda, B.E., Seetharamaiah, P., 2015. Modeling of safety-critical systems using petri nets. ACM SIGSOFT Software Engineering Notes 40, 1-7.

Swain, A.D., Guttmann, H.E., 1983. Handbook of human-reliability analysis with emphasis on nuclear power plant applications. Final report. Technical Report. Sandia National Laboratories (SNL). Albuquerque, NM, and Livermore, CA (United States). URL: http://www.osti.gov/servlets/purl/5752058/, doi:10.2172/5752058.

Talebberrouane, M., Khan, F., Lounis, Z., 2016. Availability analysis of safety critical systems using advanced fault tree and stochastic Petri net formalisms. Journal of Loss Prevention in the Process Industries 44, 193-203.

Tierney, L., Kadane, J.B., 1986. Accurate approximations for posterior moments and marginal densities. Journal of the american statistical association $81,82-86$.

Torres-Toledano, J., Sucar, L., 1998. Bayesian networks for reliability analysis of complex systems. Progress in Artificial Intelligence - IBERAMIA 98, 465-465.

Trivedi, K., Dugan, J.B., Geist, R., Smotherman, M., 1984. Hybrid reliability modeling of fault-tolerant computer systems. Computers \& electrical engineering 11, 87-108. 
Trucco, P., Cagno, E., Ruggeri, F., Grande, O., 2008. A Bayesian Belief network modelling of organisational factors in risk analysis: A case study in maritime transportation. Reliability Engineering \& System Safety 93, 845-856.

Tuncel, G., Alpan, G., 2010. Risk assessment and management for supply chain networks: A case study. Computers in industry $61,250-259$.

Ur, R.K., Zubair, M., Heo, G., 2014. Reliability analysis of nuclear I\&C architecture using Bayesian networks, in: 11th International Bhurban Conference on Applied Sciences and Technology (IBCAST), IEEE. pp. 169-174.

US Department of Defense, 1980. Procedures for Performing a Failure Mode, Effects, and Criticality Analysis (MIL-STD1629A). Technical Report. Washington DC, USA.

Vasilyev, A., Andrews, J.D., Jackson, L., Dunnett, S., 2017. Reliability modelling of PEM fuel cells with hybrid Petri nets, in: Safety and Reliability - Theory and Application: ESREL 2017. CRC Press.

Vernez, D., Buchs, D., Pierrehumbert, G., 2003. Perspectives in the use of coloured petri nets for risk analysis and accident modelling. Safety science 41, 445-463.

Vernez, D., Buchs, D.R., Pierrehumbert, G.E., Besrour, A., 2004. MORM-A Petri net based model for assessing OH\&S risks in industrial processes: Modeling qualitative aspects. Risk analysis 24, 1719-1735.

Vesely, W., Dugan, J., Fragola, J., Minarick, J., Railsback, J., 2002. Fault Tree Handbook with Aerospace Applications. Technical Report. NASA office of safety and mission assurance. Washington, DC.

Volovoi, V., 2004. Modeling of system reliability Petri nets with aging tokens. Reliability Engineering \& System Safety 84, 149-161.

Walker, M., 2009. Pandora: A Logic for the Qualitative Analysis of Temporal Fault Trees. Ph.D. thesis. University of Hull.

Wang, C., Mosleh, A., 2010. Qualitative-Quantitative Bayesian Belief Networks for reliability and risk assessment, in: Proceedings of Annual Reliability and Maintainability Symposium (RAMS), IEEE. pp. 1-5.

Wang, C.s., Xie, Y.h., 2005. A new Bayesian network model for distribution system reliability evaluation based on dual isomorphic Bayesian network model. Power system technology 7, 009.

Wang, G.y., Ma, Z.j., Hu, Q.w., 2004. The fault tree analysis based on Bayesian networks. Systems Engineering-theory \& Practice 6, 78-83

Wang, M., Lu, L., 2012. A transformation method from UML statechart to Petri nets, in: International Conference on Computer Science and Automation Engineering (CSAE), IEEE. pp. 89-92.

Wang, Y.F., Xie, M., Ng, K.M., Habibullah, M.S., 2011a. Probability analysis of offshore fire by incorporating human and organizational factor. Ocean Engineering 38, 2042-2055.

Wang, Y.F., Xie, M., Ng, K.M., Meng, Y.F., 2011b. Quantitative risk analysis model of integrating fuzzy fault tree with Bayesian network, in: International Conference on Intelligence and Security Informatics (ISI), IEEE. pp. $267-271$.

Warfield, J.N., 1973. On arranging elements of a hierarchy in graphic form. IEEE Transactions on Systems, Man, and Cybernetics , 121-132.

Weber, P., Jouffe, L., 2003. Reliability modelling with dynamic Bayesian networks. IFAC Proceedings Volumes 36, 57-62.

Weber, P., Jouffe, L., 2006. Complex system reliability modelling with dynamic object oriented Bayesian networks (DOOBN). Reliability Engineering \& System Safety 91, 149-162.

Weber, P., Medina-Oliva, G., Simon, C., Iung, B., 2012. Overview on Bayesian networks applications for dependability, risk analysis and maintenance areas. Engineering Applications of Artificial Intelligence 25, 671-682.

Weber, P., Munteanu, P., Jouffe, L., 2004. Dynamic Bayesian Networks modelling the dependability of systems with degradations and exogenous constraints. IFAC Proceedings Volumes 37, 207-212.

Wieland, C., Schmid, O., Meiler, M., Wachtel, A., Linsler, D., 2009. Reliability computing of polymer-electrolyte-membrane fuel cell stacks through petri nets. Journal of Power Sources 190, 34-39.

Williams, J., 1986. Heart-a proposed method for assessing and reducing human error, in: 9th Advances in Reliability Technology Symposium, University of Bradford.

Wilson, A.G., Huzurbazar, A.V., 2007. Bayesian networks for multilevel system reliability. Reliability Engineering \& System Safety $92,1413-1420$.

Wu, H.C., 2004. Fuzzy reliability estimation using bayesian approach. Computers \& Industrial Engineering 46, $467-493$.

Wu, H.C., 2006. Fuzzy bayesian system reliability assessment based on exponential distribution. Applied mathematical modelling 30, 509-530.

Wu, J., Yan, S., Xie, L., 2011. Reliability analysis method of a solar array by using fault tree analysis and fuzzy reasoning Petri net. Acta Astronautica 69, 960-968.

Wu, J., Yan, S., Xie, L., Gao, P., 2012. Reliability apportionment approach for spacecraft solar array using fuzzy reasoning petri net and fuzzy comprehensive evaluation. Acta Astronautica 76, 136-144.

Wu, J., Zhou, R., Xu, S., Wu, Z., 2017. Probabilistic analysis of natural gas pipeline network accident based on bayesian network. Journal of Loss Prevention in the Process Industries 46, 126-136.

Wu, W.S., Yang, C.F., Chang, J.C., Château, P.A., Chang, Y.C., 2015. Risk assessment by integrating interpretive structural modeling and Bayesian network, case of offshore pipeline project. Reliability Engineering \& System Safety 142, 515-524.

Wu, X.y., Wu, X.Y., 2015. Extended object-oriented Petri net model for mission reliability simulation of repairable PMS with common cause failures. Reliability Engineering \& System Safety 136, 109-119.

Xiaowei, Y., 2010. Common cause failure model of system reliability based on Bayesian networks. International Journal of Performability Engineering 6, 255-268.

Xiaowei, Y., Wenxue, Q., Liyang, X., 2008. A Method for System Reliabiltiy Assessment Based on Bayesian Networks. Acta Aeronautica Et Astronautica Sinica 6.

Xie, M., Wohlin, C., 1995. An additive reliability model for the analysis of modular software failure data, in: Proceedings of Sixth International Symposium onSoftware Reliability Engineering, IEEE. pp. 188-194. 
Xing, L., Shrestha, A., Dai, Y., 2011. Exact combinatorial reliability analysis of dynamic systems with sequence-dependent failures. Reliability Engineering \& System Safety 96, 1375-1385. doi:10.1016/j.ress.2011.05.007.

Yan, R., Jackson, L.M., Dunnett, S.J., 2017. Automated guided vehicle mission reliability modelling using a combined fault tree and Petri net approach. The International Journal of Advanced Manufacturing Technology , 1-13.

Yang, B.S., Jeong, S.K., Oh, Y.M., Tan, A.C.C., 2004. Case-based reasoning system with Petri nets for induction motor fault diagnosis. Expert Systems with Applications 27, 301-311.

Yang, Z., Bonsall, S., Wang, J., 2008. Fuzzy rule-based Bayesian reasoning approach for prioritization of failures in FMEA. IEEE Transactions on Reliability 57, 517-528.

Yazar, Z., 2002. A qualitative risk analysis and management tool-cramm. SANS InfoSec Reading Room White Paper .

Yazdi, M., Kabir, S., 2017. A fuzzy bayesian network approach for risk analysis in process industries. Process Safety and Environmental Protection 111, 507 - 519. doi:10.1016/j.psep.2017.08.015.

Yevkin, O., 2011. An improved modular approach for dynamic fault tree analysis, in: Proceedings of the Annual Reliability and Maintainability Symposium, IEEE. pp. 1-5.

Yin, X.w., Qian, W.x., Xie, L.y., 2008. Application of Bayesian network to reliability assessment of mechanical systems. Journal of Northeastern University Natural Science 29, 557.

Yingkui, G., Jing, L., 2012. Multi-State System Reliability: A New and Systematic Review. Procedia Engineering 29 , 531-536. doi:10.1016/j.proeng.2011.12.756.

Yongli, Z., Limin, H., Liguo, Z., Yan, W., 2008. Bayesian network based time-sequence simulation for power system reliability assessment, in: Seventh Mexican International Conference on Artificial Intelligence, IEEE. pp. 271-277.

Yontay, P., Pan, R., 2016. A computational bayesian approach to dependency assessment in system reliability. Reliability Engineering \& System Safety 152, 104-114.

Yu, D.C., Nguyen, T.C., Haddawy, P., 1999. Bayesian network model for reliability assessment of power systems. IEEE Transactions on Power Systems 14, 426-432.

Yuan, Z., Khakzad, N., Khan, F., Amyotte, P., 2015. Risk analysis of dust explosion scenarios using Bayesian networks. Risk analysis 35, 278-291.

Zafra-Cabeza, A., Ridao, M.A., Camacho, E.F., 2004. An algorithm for optimal scheduling and risk assessment of projects. Control Engineering Practice 12, 1329-1338.

Zahra, F.Z., Khouas-Oukid, S., Assoul-Semmar, Y., 2013. Dynamic bayesian networks in dynamic reliability and proposition of a generic method for dynamic reliability estimation, in: Modeling Approaches and Algorithms for Advanced Computer Applications. Springer, pp. 409-418.

Zareiee, M., Dideban, A., Orouji, A.A., 2014. Safety analysis of discrete event systems using a simplified petri net controller. ISA transactions $53,44-49$.

Zeng, R., Jiang, Y., Lin, C., Shen, X., 2012. Dependability analysis of control center networks in smart grid using stochastic Petri nets. IEEE Transactions on Parallel and Distributed Systems 23, 1721-1730.

Zhang, D., Yan, X., Yang, Z.L., Wall, A., Wang, J., 2013. Incorporation of formal safety assessment and Bayesian network in navigational risk estimation of the Yangtze river. Reliability Engineering \& System Safety 118, 93-105.

Zhang, L., Wu, X., Skibniewski, M.J., Zhong, J., Lu, Y., 2014. Bayesian-network-based safety risk analysis in construction projects. Reliability Engineering \& System Safety 131, 29-39.

Zhang, X., Miao, Q., Fan, X., Wang, D., 2009. Dynamic fault tree analysis based on Petri nets, in: 8th International Conference on Reliability, Maintainability and Safety(ICRMS), IEEE, Chengdu. pp. 138-142.

Zhang, Y., Zhang, Y., Wen, F., Chung, C.Y., Tseng, C.L., Zhang, X., Zeng, F., Yuan, Y., 2016. A fuzzy petri net based approach for fault diagnosis in power systems considering temporal constraints. International Journal of Electrical Power \& Energy Systems 78, 215-224.

Zhao, L., Wang, X., Qian, Y., 2012. Analysis of factors that influence hazardous material transportation accidents based on bayesian networks: A case study in china. Safety science 50, 1049-1055.

Zhou, J., Reniers, G., Zhang, L., 2017. A weighted fuzzy Petri-net based approach for security risk assessment in the chemical industry. Chemical Engineering Science 174, 136-145.

Zhou, K.Q., Zain, A.M., 2016. Fuzzy petri nets and industrial applications: a review. Artificial Intelligence Review 45 , $405-446$.

Zhou, S., Qin, Z., Zhang, F., Zhang, X., Chen, W., Liu, J., 2003. Colored Petri net based attack modeling. Rough Sets, Fuzzy Sets, Data Mining, and Granular Computing, 583-583.

Zhou, Z., Jin, G., Dong, D., Zhou, J., 2006a. Reliability analysis of multistate systems based on Bayesian networks, in: 13th Annual IEEE International Symposium and Workshop on Engineering of Computer Based Systems, IEEE. pp. 1-6.

Zhou, Z.b., Dong, D.d., Zhou, J.l., 2006b. Application of Bayesian networks in reliability analysis. Systems Engineering-Theory \& Practice 6, 95-100.

Zio, E., 2009. Reliability engineering: Old problems and new challenges. Reliability Engineering \& System Safety $94,125-141$. doi:10.1016/j.ress.2008.06.002.

Zurawski, R., Zhou, M., 1994. Petri nets and industrial applications: A tutorial. IEEE Transactions on industrial electronics 41, 567-583. 